\title{
GENERIC CYCLES, LEFSCHETZ REPRESENTATIONS, AND THE GENERALIZED HODGE AND BLOCH CONJECTURES FOR ABELIAN VARIETIES
}

\author{
CHARLES VIAL
}

\begin{abstract}
We prove Bloch's conjecture for correspondences on powers of complex abelian varieties, that are "generically defined". As an application we establish vanishing results for (skew-)symmetric cycles on powers of abelian varieties and we address a question of Voisin concerning (skew-)symmetric cycles on powers of K3 surfaces in the case of Kummer surfaces. We also prove Bloch's conjecture in the following situation. Let $\gamma$ be a correspondence between two abelian varieties $A$ and $B$ that can be written as a linear combination of products of symmetric divisors. Assume that $A$ is isogenous to the product of an abelian variety of totally real type with the power of an abelian surface. We show that $\gamma$ satisfies the conclusion of Bloch's conjecture. A key ingredient consists in establishing a strong form of the generalized Hodge conjecture for Hodge sub-structures of the cohomology of $A$ that arise as sub-representations of the Lefschetz group of $A$. As a by-product of our method, we use a strong form of the generalized Hodge conjecture established for powers of abelian surfaces to show that every finite-order symplectic automorphism of a generalized Kummer variety acts as the identity on the zero-cycles.
\end{abstract}

\section{INTRODUCTION}

Throughout this note, Chow groups are with rational coefficients. Let $X$ be a smooth projective complex variety of dimension $d$ and let $\gamma$ be a correspondence in $\mathrm{CH}^{d}(X \times X)$ such that $\gamma_{*} \mathrm{CH}_{0}(X)=0$. The Bloch-Srinivas argument [14] implies that $\gamma^{*} \mathrm{H}^{*}(X, \mathbb{Q})$ is supported on a divisor, which in turn implies that $\gamma^{*} \mathrm{H}^{i, 0}(X)=0$ for all integers $i$. The Bloch conjecture stipulates that, conversely, should $\gamma^{*} \mathrm{H}^{i, 0}(X)$ vanish for all integers $i$, then $\gamma$ acts nilpotently on $\mathrm{CH}_{0}(X)$. (In fact, the conjecture predicts that $\gamma$ should act as zero on the graded pieces of the conjectural Bloch-Beilinson filtration on $\mathrm{CH}_{0}(X)$.)

More generally, if $\gamma_{*} \mathrm{CH}_{r}(X)=0$ for all $r<n$, then the Bloch-Srinivas argument implies that $\gamma^{*} \mathrm{H}^{*}(X, \mathbb{Q})$ is supported on a subscheme of codimension $n$, which in turn implies that $\gamma^{*} H^{i, j}(X)=0$ for all integers $i$ and $j<n$. The generalized Bloch conjecture is the following converse assertion:

Conjecture 1 (Generalized Bloch conjecture). Let $X$ be a smooth projective complex variety of dimension $d$, and let $\gamma \in \mathrm{CH}^{d}(X \times X)$ be a correspondence. Suppose that $\gamma^{*} \mathrm{H}^{i, j}(X)=0$ for all $j<n$, or, equivalently in terms of the Hodge coniveau filtration, $\gamma^{*} \mathrm{H}^{*}(X, \mathbb{Q}) \subseteq \mathrm{N}_{H}^{n} \mathrm{H}^{*}(X, \mathbb{Q})$. Then $\gamma_{*}$ acts nilpotently on $\mathrm{CH}_{r}(X)$ for all $r<n$.

The conjecture is wide open, but has notably been established for surfaces with $\mathrm{H}^{2,0}=0$ not of general type [13], for certain surfaces with $\mathrm{H}^{2,0}=0$ of general type [52, 55], and for finite-order symplectic automorphisms of K3 surfaces [54, 28].

Conjecture 1 follows from the combination of (a) the generalized Hodge conjecture (for smooth projective varieties, and not just for $X$ ) and (b) the existence of the conjectural Bloch-Beilinson filtration. Indeed, if $\gamma^{*} \mathrm{H}^{i, j}(X)=0$ for all $j<n$, then the generalized Hodge conjecture for $X$ implies that $\gamma^{*} \mathrm{H}^{*}(X, \mathbb{Q})$ is supported on a closed subscheme $X$ of codimension $n$. By [7], the

2010 Mathematics Subject Classification. 14C25, 14C15, 14C30, 14K10.

Key words and phrases. Algebraic cycles, Abelian varieties, Motives, Chow groups, Bloch-Beilinson filtration, Bloch conjecture, Lefschetz group, generalized Hodge conjecture, generalized Kummer varieties, symplectomorphisms. 
standard conjectures (for $\tilde{Z} \times X$, where $\tilde{Z} \rightarrow Z$ is a desingularization) then provide a selfcorrespondence $p \in \mathrm{CH}^{d}(X \times X)$ supported on $Z \times X$ such that $p$ induces in cohomology a projector with image $\gamma^{*} \mathrm{H}^{*}(X, \mathbb{Q})$ (see Conjecture 1.6). It follows that $\gamma \circ\left(\Delta_{X}-p\right)$ acts trivially on $\mathrm{H}^{*}(X, \mathbb{Q})$, i.e., that $\gamma \circ\left(\Delta_{X}-p\right)$ is homologically trivial. In particular, if $\mathrm{F}^{\bullet}$ denotes the conjectural Bloch-Beilinson filtration, $\gamma_{*} \circ\left(\Delta_{X}-p\right)_{*}$ sends $\mathrm{F}^{l} \mathrm{CH}_{r}(X)$ to $\mathrm{F}^{l+1} \mathrm{CH}_{r}(X)$ for all $l$ and all $r$. Since conjecturally $\mathrm{F}^{r+1} \mathrm{CH}_{r}(X)=0$ for all $r$, we find that $\gamma_{*} \circ\left(\Delta_{X}-p\right)_{*}$ is nilpotent. (Alternately, the conjectural Kimura-O'Sullivan finiteness for $X$ implies that $\gamma \circ\left(\Delta_{X}-p\right)$ is nilpotent). Finally, for support reasons, $p_{*}$ acts as zero on $\mathrm{CH}_{r}(X)$ for $r<n$, and we conclude that $\gamma_{*}$ acts nilpotently on $\mathrm{CH}_{r}(X)$ for $r<n$.

We note that by applying Conjecture 1 to $\gamma=\Delta_{X}-\sum_{i} \pi_{\text {alg }}^{2 i}$, where the $\pi_{\text {alg }}^{2 i}$ are projectors on the degree- $2 i$ Hodge classes (which conjecturally exist), one recovers the more classical version of the generalized Bloch conjecture stated e.g. in [56, Conj. 1.9]. We also note that one cannot conclude in general that $\gamma_{*}$ acts as zero on $\mathrm{CH}_{r}(X)$ for $r<n$. Consider indeed a smooth projective curve $C$ of positive genus and the correspondence $\gamma=C \times \alpha$, where $\alpha$ is a non-zero degree-0 0-cycle on $C$; then $\gamma^{*} \mathrm{H}^{*}(C, \mathbb{Q})=0$ and $(\gamma \circ \gamma)_{*} \mathrm{CH}_{0}(C)=0$, but $\gamma_{*} \mathrm{CH}_{0}(C)=\mathbb{Q} \alpha \neq 0$.

Our main results are Theorem 2.15 and Theorem 3.14. We establish the generalized Bloch conjecture for certain correspondences between abelian varieties, that are of two types: either "generically defined", or belong to the sub-algebra generated by symmetric divisors (with some further assumptions on the abelian varieties). In both cases, the strategy consists in first showing that the Hodge sub-structure $\gamma^{*} \mathrm{H}^{*}(X, \mathbb{Q})$ is supported in codimension $n$ in a strong sense (existence of a cycle $p \in \mathrm{CH}^{d}(X \times X)$ as in the discussion above with additional properties), in particular that the generalized Hodge conjecture for $\gamma^{*} \mathrm{H}^{*}(X, \mathbb{Q})$ holds; see Propositions 2.13 and 3.12. For that matter, we formulate in Conjecture 1.6 a strong (but equivalent, when considered for all complex smooth projective varieties) version of the generalized Hodge conjecture. This information on the cohomological support of $\gamma$ is then lifted to rational equivalence thanks either to Kimura-O'Sullivan finite-dimensionality (Theorem 1.1) or to a recent result of O'Sullivan (Theorem 1.2). In the latter case, that is, when $\gamma$ is in addition symmetrically distinguished (see §1.2), then one can conclude that $\gamma_{*} \mathrm{CH}_{r}(X)=0$ for all $r<n$ (see Theorems 2.15(2) and 3.14).

0.1. Generically defined cycles. A generically defined cycle on the $m$-fold power of a polarized complex abelian variety $A$ of degree $d^{2}$ and dimension $g$ is a cycle (with rational coefficients) in $\mathrm{CH}^{*}\left(A^{m}\right)$ that is the restriction, for some integer $N \geq 3$ (in fact, by Remark 2.2, for any integer $N \geq 3$ ), of a cycle on the $m$-fold power of the universal polarized abelian variety of degree $d^{2}$ and dimension $g$ with level- $N$ structure; see Definition 2.1. A generically defined self-correspondence on the $m$-fold power of complex polarized abelian varieties of degree $d^{2}$ and dimension $g$ is a generically defined cycle of codimension $m g$ on the $2 m$-fold power of polarized complex abelian varieties of degree $d^{2}$ and of dimension $g$.

Our first main result is Theorem 2.15, a special instance of which is the following:

Theorem 1. Suppose that $\gamma$ is a generically defined correspondence on the $m$-fold power of polarized complex abelian varieties. Assume that $\gamma^{*} \mathrm{H}^{i, j}\left(A^{m}\right)=0$ for all $j<n$ for some (equivalently, for all) polarized complex abelian variety $A$ of dimension $g$ and degree $d^{2}$. Then $\gamma_{*}$ acts nilpotently on $\mathrm{CH}_{r}\left(A^{m}\right)$ for all $r<n$.

The proof consists in first establishing Theorem 1 for a very general complex abelian variety $A$. For such a variety, a strong form of the generalized Hodge conjecture (as in Conjecture 1.6) holds (Hazama's Theorem 2.12) and makes it possible to interpret the $n$-th Hodge coniveau part (see Definition 1.4) $\mathrm{N}_{H}^{n} \mathrm{H}^{*}\left(A^{m}, \mathbb{Q}\right)$ as a "generically defined" sub-motive of $A^{m}$ whose Tate twist by $n$ is effective and, in fact, isomorphic to a direct summand of a finite direct sum of motives of $A^{m}$; see the key Proposition 2.13. One can conclude by using the Kimura finite-dimensionality [29] of motives of abelian varieties. One establishes Theorem 1 for all abelian varieties by specialization. 
Recall from Beauville [11] that the Chow group of zero-cycles on an abelian variety $A$ of dimension $g$ splits into eigenspaces as

$$
\mathrm{CH}_{0}(A)=\mathrm{CH}_{0}(A)_{(0)} \oplus \cdots \oplus \mathrm{CH}_{0}(A)_{(g)},
$$

where $\mathrm{CH}_{0}(A)_{(i)}=\left\{a \in \mathrm{CH}_{0}(A):[n]_{*} a=n^{i} a\right.$ for all $\left.n \in \mathbb{Z}\right\}$ with $[n]: A \rightarrow A$ the multiplication-by- $n$ homomorphism. As an application of Theorem 1 , we obtain:

Corollary 1 (Theorem 4.1). Let $A$ be an abelian variety of dimension $g$, and let $i$ be a nonnegative integer. Let $N>\left(\begin{array}{l}g \\ i\end{array}\right)$ and suppose that $a_{j}, 1 \leq j \leq N$, are zero-cycles on $A$ such that $[n]_{*} a_{j}=n^{i} a_{j}$ for all integers $n$. Then the following holds.

- For $i$ odd, the symmetrization of $a_{1} \times \cdots \times a_{N}$ vanishes, i.e.

$$
\sum_{\sigma \in \mathfrak{S}_{N}} a_{\sigma(1)} \times \cdots \times a_{\sigma(N)}=0 \quad \text { in } \mathrm{CH}_{0}\left(A^{N}\right) .
$$

- For $i$ even, the anti-symmetrization of $a_{1} \times \cdots \times a_{N}$ vanishes, i.e.

$$
\sum_{\sigma \in \mathfrak{S}_{N}} \operatorname{sgn}(\sigma) a_{\sigma(1)} \times \cdots \times a_{\sigma(N)}=0 \quad \text { in } \mathrm{CH}_{0}\left(A^{N}\right) .
$$

0.2. Lefschetz sub-representations. Let $A$ be an abelian variety. We define

$$
\mathrm{R}^{*}(A) \subseteq \mathrm{CH}^{*}(A)
$$

to be the sub-algebra of $\mathrm{CH}^{*}(A)$ generated by symmetric divisors. Note that if $B$ is another abelian variety, then the class of the graph of any homomorphism $A \rightarrow B$ belongs to $\mathrm{R}^{*}(A \times B)$ (see Proposition 3.11). As a link to Theorem 1, we note that all generically defined cycles on the $m$-fold power of an abelian variety $A$ that we consider in explicit examples belong to $\mathrm{R}^{*}\left(A^{m}\right)$; see however Question 2.8. We can prove the generalized Bloch conjecture for correspondences that belong to $\mathrm{R}^{*}$ on certain abelian varieties (which are not necessarily very general).

Definition 1 (Abelian varieties of totally real type). An abelian variety $A$ is said to be of totally real type if the center of its endomorphism ring $\operatorname{End}^{0}(A):=\operatorname{End}(A) \otimes_{\mathbb{Z}} \mathbb{Q}$ is isomorphic to a product of totally real fields. Equivalently, $A$ is of totally real type if it is isogenous to $A_{1}^{m_{1}} \times \cdots \times A_{s}^{m_{s}}$ with the $A_{i}$ simple of type I, II, or III (see §3.2).

Our second main result is Theorem 3.14, a special instance of which is the following:

Theorem 2. Let $A$ and $B$ be two abelian varieties, and let $\gamma$ be a cycle in $\mathrm{R}^{*}(A \times B)$. Suppose that $A$ is of totally real type. If $\gamma^{*} \mathrm{H}^{i, j}(B)=0$ for all $j<n$, then $\gamma_{*} \mathrm{CH}_{r}(A)=0$ for all $r<n$.

There are two main arguments entering the proof of Theorem 2. First, as explained in $\S 3.1$, the fact that $\gamma$ belongs to $\mathrm{R}^{*}(A \times B)$ implies that the Hodge sub-structure $\gamma^{*} \mathrm{H}^{*}(B, \mathbb{Q})$ is a subrepresentation of the Lefschetz group of $A$ acting on $\mathrm{H}^{*}(A, \mathbb{Q})$. The first step does not consist in establishing the generalized Hodge conjecture for $A$ but, instead, consists in showing that any sub-representation of the Lefschetz group of $A$ acting on $\mathrm{H}^{*}(A, \mathbb{Q})$ satisfies a strong form of the generalized Hodge conjecture (as in Conjecture 1.6); see Proposition 3.12. We note that if $A$ is a very general complex abelian variety, then $\operatorname{End}^{0}(A)=\mathbb{Q}$ and, by coincidence of the Lefschetz group of $A$ with its Hodge group, every Hodge sub-structure of $\mathrm{H}^{*}\left(A^{m}, \mathbb{Q}\right)$ is a Lefschetz subrepresentation. The generalized Hodge conjecture for self-powers of the very general complex abelian variety was established by Hazama [26] (see Theorem 2.12). By shifting our attention to Lefschetz sub-representations, we can generalize the aforementioned result of Hazama (we refer to Theorem 3.7 for a more precise statement):

Theorem 3 (strong GHC for Lefschetz sub-representations of abelian varieties of totally real type). Let $A$ be a complex abelian variety, and let $H \subseteq \mathrm{H}^{k}(A, \mathbb{Q})$ be a Lefschetz sub-representation of Hodge level $\leq k-2 n$. Suppose that $A$ is of totally real type. Then $H$ satisfies the strong generalized Hodge conjecture 1.6, in particular, $H$ is supported on a closed subset of codimension $n$. 
In the second step, instead of using Kimura's finite-dimensionality which would only yield that $\gamma$ acts nilpotently on $\mathrm{CH}_{r}(A)$ for all $r<n$, we utilize a recent powerful result of O'Sullivan [41] which in particular implies that the ring $\mathrm{R}^{*}(A)$ injects into cohomology for all abelian varieties $A$. We refer to the proof of Theorem 3.14 for the details.

Unfortunately, our method for establishing (a strong form of) the generalized Hodge conjecture for Lefschetz sub-representations of $\mathrm{H}^{i}\left(A^{m}, \mathbb{Q}\right)$ for $A$ of totally real type does not seem to extend in a direct way to the interesting case of abelian varieties of type IV or even to that of abelian varieties of CM type; see Remark 3.9. As far as we know, the conjecture is still open for the product of four pairwise non-isogenous CM elliptic curves.

Nonetheless, the generalized Hodge conjecture was established by Abdulali [1] for powers of a simple abelian surface of CM type (see Theorem 3.10). Abdulali's proof yields a strong form of the generalized Hodge conjecture (as in Conjecture 1.6) for powers of abelian varieties of dimension $\leq 2$ (see Corollary 3.13). Using Abdulali's theorem, we establish in Theorem 3.14 a slightly more general version of Theorem 2 by allowing $A$ to be isogenous to the product of an abelian variety of totally real type with either the power of a CM abelian surface or a product of powers of three CM elliptic curves. Again the key input consists in establishing a strong form of the generalized Hodge conjecture for Lefschetz sub-representations (Proposition 3.12). Since the case of powers of abelian surfaces is particularly telling due to the link with so-called generalized Kummer varieties, we single out the following statement from Theorem 3.14:

Theorem 4. Let $A$ and $B$ be two abelian varieties, and let $\gamma$ be a cycle in $\mathrm{R}^{*}(A \times B)$. Suppose that $A$ is isogenous to a power of an abelian variety of dimension $\leq 2$. If $\gamma^{*} \mathrm{H}^{i, j}(B)=0$ for all $j<n$, then $\gamma_{*} \mathrm{CH}_{r}(A)=0$ for all $r<n$.

0.3. Applications. Section 4 is concerned with concrete applications of the above results. Specifically, Theorems 4.1, 4.3 and 4.4 provide vanishing results for (skew)-symmetric cycles on powers of abelian varieties and generalized Kummer varieties, while Theorem 4.6 settles a conjecture of Voisin about K3 surfaces in the case of Kummer surfaces. All these results are proved as consequences of Theorem 1, so that the reader interested only in those can skip reading Section 3 entirely. Finally, in $\S 4.4$ we establish a variant of Theorem 4 (Theorem 4.7) which we use in $\S 4.5$ to show that a finite-order symplectic automorphism of a generalized Kummer variety acts as the identity on the Chow group of zero-cycles (see also Proposition 4.11) :

Theorem 5 (Theorem 4.10). Let $A$ be an abelian surface. If $f$ is a finite-order symplectic automorphism of the generalized Kummer variety $K_{n}(A)$, then $f_{*}: \mathrm{CH}_{0}\left(K_{n}(A)\right) \rightarrow \mathrm{CH}_{0}\left(K_{n}(A)\right)$ is the identity map.

Acknowledgments. I would like to thank Robert Laterveer for bringing to my attention the questions posed by Voisin in [53, §3], and Giuseppe Ancona for very useful discussions.

\section{Preliminaries}

1.1. Polarized abelian varieties, and level structures. A polarization $L$ on a complex abelian variety $A$ of dimension $g$ is by definition the first Chern class of an ample line bundle $\mathcal{L}$. We denote $\hat{A}=\operatorname{Pic}^{0}(A)$ the dual abelian variety and $\mathcal{P}_{A}$ the Poincaré line-bundle on $A \times \hat{A}$. Let $\phi_{\mathcal{L}}: A \rightarrow \hat{A}$ be the morphism given on points by $a \mapsto t_{a}^{*} \mathcal{L} \otimes \mathcal{L}^{-1}$. By definition, the degree of the polarization $L$ is the degree of the isogeny $\phi_{\mathcal{L}}: A \rightarrow \hat{A}$; it is a square since we have $\operatorname{deg}(L)=\chi(\mathcal{L})^{2}$. We will often view the Poincaré line-bundle $\mathcal{P}_{A}$ as a line-bundle on $A \times A$, by pulling back along id $\operatorname{id}_{A} \times \phi_{\mathcal{L}}$ the Poincaré line-bundle on $A \times \hat{A}$. The Fourier-Mukai transform of $\mathcal{L}$ is the sheaf $\mathcal{F}(\mathcal{L}):=p_{2, *}\left(\mathcal{P}_{A} \otimes p_{1}^{*} \mathcal{L}\right)$; it is a vector-bundle on $\hat{A}$. The dual polarization $\hat{L}$ on $\hat{A}$ is the first Chern class of $\operatorname{det}(\mathcal{F}(\mathcal{L}))^{-1} ;$ see $[12]$.

Denote $\iota_{\Delta}: A \rightarrow A \times A$ the diagonal embedding. We define a correspondence $\Lambda_{A}^{i}$ in $\mathrm{CH}_{i}(A \times$ A) as follows :

$$
\Lambda_{A}^{i}= \begin{cases}\iota_{\Delta, *} L^{g-i} & \text { if } i \leq g \\ \hat{\mathcal{F}} \circ\left(\iota_{\Delta, *} \hat{L}^{i-g}\right) \circ \mathcal{F} & \text { if } i>g .\end{cases}
$$


Note that $\Lambda_{A}^{i}$ induces an isomorphism $\mathrm{H}^{i}(A, \mathbb{Q}) \stackrel{\simeq}{\longrightarrow} \mathrm{H}^{2 g-i}(A, \mathbb{Q})$.

Following [38, §6], for a projective abelian scheme $\mathcal{A} \rightarrow S$ over a Noetherian scheme $S$, we define its dual $\hat{\mathcal{A}} \rightarrow S$ to be the projective scheme that is the open sub-group-scheme of $\operatorname{Pic}(\mathcal{A} / S)$ whose geometric points correspond to the invertible sheaves some power of which are algebraically equivalent to zero, and we define a polarization on $\hat{\mathcal{A}} \rightarrow S$ to be a $S$-homomorphism $\mathcal{A} \rightarrow \hat{\mathcal{A}}$ such that, for all geometric points $\bar{s}$ of $S$, the induced $\mathcal{A}_{\bar{s}} \rightarrow \hat{\mathcal{A}}_{\bar{s}}$ is of the form $\phi_{\mathcal{L}}$ for some ample line-bundle $\mathcal{L}$ on $\mathcal{A}_{\bar{s}}$.

Let $\mathcal{A} \rightarrow S$ be a projective abelian scheme of relative dimension $g$ over a Noetherian scheme $S$, and let $N$ be an integer $\geq 2$. Assume that the characteristics of the residue fields of all closed points of $S$ do not divide $N$. A level- $N$ structure on $\mathcal{A} \rightarrow S$ consists of $2 g$ sections $\sigma_{1}, \ldots, \sigma_{2 g}$ of $\mathcal{A} \rightarrow S$ such that their restriction to any geometric point $\bar{s}$ of $S$ provide a basis of the $N$-torsion of the fiber of $\mathcal{A} \rightarrow S$ over $\bar{s}$, and such that $[N] \circ \sigma_{i}=0_{\mathcal{A}}$ for all $i$, where $[N]$ denotes the multiplication-by- $N$ morphism and where $0_{\mathcal{A}}$ is the identity section of $\mathcal{A} \rightarrow S$.

1.2. Motives of abelian varieties, symmetrically distinguished cycles. We will use freely the language of Chow motives, as is described for instance in [8]. The unit motive is denoted $\mathbb{1}$ and the motive of a smooth projective variety is denoted $\mathfrak{h}(X)$. Our convention for the Tate twist is such that $\mathfrak{h}\left(\mathbb{P}^{1}\right)=\mathbb{1} \oplus \mathbb{1}(-1)$.

The Chow motives of abelian varieties have particularly nice properties. First they are finitedimensional in the sense of Kimura [29]. Without going into the details of Kimura's notion of finite-dimensionality, let us only mention the following property:

Theorem 1.1 (Kimura [29]). Let $A$ be a complex abelian variety of dimension g, and let $\Gamma \in$ $\mathrm{CH}^{g}(A \times A)$ be a self-correspondence on $A$. Assume that $\Gamma$ is numerically trivial. Then $\Gamma$ is nilpotent, i.e., there exists a positive integer $N$ such that $\Gamma^{\circ N}=0$ in $\mathrm{CH}^{g}(A \times A)$.

Second, O'Sullivan [41] has recently identified a sub-algebra of $\mathrm{CH}^{*}(A)$ consisting of cycles that are called symmetrically distinguished (see [41, p.2] for a definition), with the following property:

Theorem 1.2 (O'Sullivan [41]). Let $A$ be a complex abelian variety. The symmetrically distinguished cycles in $\mathrm{CH}^{*}(A)$ form a graded $\mathbb{Q}$-sub-algebra, denoted $\mathrm{DCH}^{*}(A)$, that contains symmetric divisors and that is stable under pull-backs and push-forwards along homomorphisms of abelian varieties. Moreover the composition

$$
\mathrm{DCH}^{*}(A) \hookrightarrow \mathrm{CH}^{*}(A) \rightarrow \overline{\mathrm{CH}}^{*}(A)
$$

is an isomorphism of $\mathbb{Q}$-algebras. Here, $\overline{\mathrm{CH}}^{*}(A)$ denotes the Chow ring of $A$ modulo numerical equivalence. In particular, a symmetrically distinguished cycle that is homologically trivial is rationally trivial.

The following definition will be relevant to our work concerned with Lefschetz representations; see e.g. Lemma 3.4.

Definition 1.3. For a complex abelian variety $A$, we denote

$$
\mathrm{R}^{*}(A) \subset \mathrm{CH}^{*}(A)
$$

the $\mathbb{Q}$-sub-algebra generated by symmetric divisors and we denote $\overline{\mathrm{R}}^{*}(A)$ its image in $\overline{\mathrm{CH}}^{*}(A)$, or equivalently, since homological and numerical equivalence agree on complex abelian varieties, its image in $\mathrm{H}^{*}(A, \mathbb{Q})$ under the cycle class map.

By O'Sullivan's Theorem 1.2, $\mathrm{R}^{*}(A)$ is a sub-algebra of $\mathrm{DCH}^{*}(A)$ that maps isomorphically onto $\overline{\mathrm{R}}^{*}(A)$ via the cycle class map ${ }^{1}$. Note that a polarization of $A$ is a symmetric divisor on $A$, and that the first Chern class of the Poincaré line-bundle is a symmetric divisor on $A \times \hat{A}$. We note that by Proposition 3.11 below the cycles $\Lambda_{A}^{i}$ of (1) belong to $\mathrm{R}^{*}(A \times A)$.

\footnotetext{
${ }^{1}$ That $\mathrm{R}^{*}(A)$ maps isomorphically onto $\overline{\mathrm{R}}^{*}(A)$ was also established independently by Ancona [5] and Moonen [37].
} 
1.3. Hodge structures and the generalized Hodge conjecture. A Q-Hodge structure $H$ is a rational vector space of finite dimension together with a decomposition of $H_{\mathbb{C}}:=H \otimes_{\mathbb{Q}} \mathbb{C}$ as a direct sum of complex linear subspaces $H^{p, q}$ for integers $p, q$ such that $\overline{H^{p, q}}=H^{q, p}$ and such that the grading by $p+q$, called the weight grading, is defined over $\mathbb{Q}$. The level of a Hodge structure $H$ is defined as

$$
\ell(H):=\max \left\{|p-q|: H^{p, q} \neq 0\right\},
$$

with the convention that we declare $H=0$ to have level $-\infty$. A Hodge structure $H$ is said to be effective if $H^{p, q}=0$ for $p<0$.

Definition 1.4. Let $H$ be a rational Hodge structure of weight $k$. The Hodge coniveau filtration is

$$
\mathrm{N}_{H}^{n} H=\text { the largest Hodge sub-structure of } H \text { of level } \leq k-2 n .
$$

In other words, $\mathrm{N}_{H}^{n} H$ is the largest Hodge sub-structure $H^{\prime}$ of $H$ such that $H^{\prime} \otimes \mathbb{Q}(n)$ is effective. Here $\mathbb{Q}(n)$ denotes the 1-dimensional Hodge structure of weight $-2 n$ and level 0 .

Conjecture 1.5 (Grothendieck's generalized Hodge conjecture). Let $X$ be a complex smooth projective variety. If $H$ is a sub-Hodge structure of $\mathrm{H}^{k}(X, \mathbb{Q})$ of level $\leq k-2 n$, i.e. $H \subseteq$ $\mathrm{N}_{H}^{n} \mathrm{H}^{k}(X, \mathbb{Q})$, then $H$ is supported in codimension $n$, i.e. there exists a closed subscheme $Z \subseteq X$ of codimension $n$ such that $H$ is mapped to zero under the restriction homomorphism $\mathrm{H}^{k}(X, \mathbb{Q}) \rightarrow \mathrm{H}^{k}(X \backslash Z, \mathbb{Q})$.

Combining the above with the standard conjectures, a theorem of Yves André [7] on motivated cycles allows us to formulate the following conjecture (see e.g. the proof of [4, Prop. 4.1]).

Conjecture 1.6 (Strong form of the generalized Hodge conjecture). Let $X$ be a complex smooth projective variety of dimension d. If $H$ is a sub-Hodge structure of $\mathrm{H}^{k}(X, \mathbb{Q})$ of level $\leq k-2 n$, i.e. $H \subseteq \mathrm{N}_{H}^{n} \mathrm{H}^{k}(X, \mathbb{Q})$, then there exists a closed subscheme $Z \subseteq X$ of codimension $n$ and $a$ correspondence $p \in \mathrm{CH}^{d}(X \times X)$ supported on $Z \times X$ such that $p^{*}: \mathrm{H}^{*}(X, \mathbb{Q}) \rightarrow \mathrm{H}^{*}(X, \mathbb{Q})$ is an idempotent with image $H$.

We note that the standard conjectures are implied by the (generalized) Hodge conjecture. Therefore, the generalized Hodge conjecture 1.5 for all complex smooth projective varieties implies the validity of Conjecture 1.6. In particular, Conjectures 1.5 and 1.6 are equivalent when considered for all complex smooth projective varieties. This stronger formulation of the generalized Hodge conjecture will be crucial to our main results; see Propositions 2.13 and 3.12.

\section{Generically Defined CyCles}

2.1. Generically defined cycles on self-products of abelian varieties. A fundamental result of Grothendieck and Mumford [38, Theorem 7.9] is that, for $N \geq 3$, the fine moduli scheme $\mathcal{A}_{g, d, N}$ for polarized abelian varieties of degree $d^{2}$ and dimension $g$ with level- $N$ structure exists, and that it is moreover quasi-projective over $\operatorname{Spec} \mathbb{Z}$.

Definition 2.1 (Generically defined cycles on abelian varieties). Let $m, g$ and $d$ be positive integers. A generically defined cycle on the $m$-fold power of a polarized complex abelian variety $A$ of degree $d^{2}$ and dimension $g$ is a cycle in $\mathrm{CH}^{*}\left(A^{m}\right)$ that is the restriction, for some integer $N \geq 3$, of a cycle on the $m$-fold power of the universal polarized abelian variety of degree $d^{2}$ and dimension $g$ with level- $N$ structure.

For the sake of this paper we only consider cycles with rational coefficients, but of course the definition of generically defined cycles on abelian varieties makes sense for Chow groups with integral coefficients. However, with rational coefficients, the definition is independent of the choice of a level structure:

Remark 2.2. By considering the natural finite étale morphism $\mathcal{A}_{g, d, M} \rightarrow \mathcal{A}_{g, d, N}$ for integers $M, N \geq 3$ such that $N$ divides $M$, we see that generically defined cycles on the $m$-fold power of a polarized complex abelian variety $A$ are in fact the restriction, for all integers $N \geq 3$, of a cycle 
on the $m$-fold power of the universal polarized abelian variety of degree $d^{2}$ and dimension $g$ with level- $N$ structure. In particular, generically defined cycles on the $m$-fold power of a polarized complex abelian variety $A$ form a $\mathbb{Q}$-sub-algebra of $\mathrm{CH}^{*}\left(A^{m}\right)$.

Remark 2.3 (Universally defined cycles on abelian varieties). In our applications, the generically defined cycles that we are going to consider will actually satisfy the following stronger condition. Let $m$ and $g$ be nonnegative integers. A universally defined cycle on the $m$-fold power of polarized abelian varieties of dimension $g$ consists, for every polarized abelian scheme $\mathcal{A} \rightarrow B$ of relative dimension $g$ over a smooth quasi-projective complex variety $B$, of a cycle $z_{\mathcal{A}} \in \mathrm{CH}^{*}\left(\mathcal{A}_{/ B}^{m}\right)$ such that for every morphism $f: B^{\prime} \rightarrow B$ of smooth quasi-projective complex varieties $z_{\mathcal{A}}$ restricts to $z_{\mathcal{A} \times{ }_{B} B^{\prime}}$ under the natural morphism $\left(\mathcal{A} \times{ }_{B} B^{\prime}\right)_{/ B^{\prime}}^{m} \rightarrow \mathcal{A}_{/ B}^{m}$. Here the abelian scheme $\mathcal{A} \times{ }_{B} B^{\prime} \rightarrow B^{\prime}$ is understood to be equipped with the polarization induced by that of $\mathcal{A}$.

Remark 2.4. It is clear that, when restricted to the $m n$-fold powers of polarized abelian varieties of dimension $g$, a cycle that is generically defined for $m$-fold powers of polarized abelian varieties of dimension $n g$ is generically defined for $m n$-fold powers of polarized abelian varieties of dimension $g$.

Example 2.5. The polarization of a polarized abelian variety is generically defined. Likewise, the first Chern class of the Poincaré line-bundle (see §1.1) and the correspondences $\Lambda_{A}^{i}$ of (1) are generically defined on 2-fold products of polarized abelian varieties.

For future use, let us give the following examples of generically defined self-correspondences on abelian varieties:

Lemma 2.6. Suppose that $(A, L)$ is a polarized complex abelian variety of dimension $g$. Then there exist, for all integers $k$ and $n$, idempotent correspondences $p^{k, n} \in \mathrm{DCH}^{g}(A \times A)$ that are generically defined for 2 -fold products of abelian varieties, and whose action in cohomology are the orthogonal projectors

$$
p^{k, n}: \mathrm{H}^{*}(A, \mathbb{Q}) \rightarrow L^{n} \mathrm{H}^{k-2 n}(A, \mathbb{Q})_{\operatorname{prim}} \rightarrow \mathrm{H}^{*}(A, \mathbb{Q}) .
$$

In particular, the Chow-Künneth projectors $\pi_{A}^{k}:=\sum_{n} p^{k, n}$ are generically defined.

Proof. Kleiman [30, Proposition 2.3] showed that the orthogonal projectors $p^{k, n}$ are algebraic for all smooth projective complex varieties that satisfy Grothendieck's Lefschetz standard conjecture. In fact, given a polarized abelian variety $(A, L)$ it is shown in [30, Proposition 1.4.4] that the projectors $p^{k, n}$ are the classes of cycles (denoted abusively also $p^{k, n}$ ) that belong to the sub-algebra of $\mathrm{CH}^{*}(A \times A)$ generated by the $\Lambda_{A}^{i}$ for $0 \leq i \leq 2 g$ (see (1)). Since the cycles $\Lambda_{A}^{i}$ are generically defined for 2-fold products of abelian varieties (Example 2.5), so are the cycles $p^{k, n}$. Finally, note that the cycles $\Lambda_{A}^{i}$ belong to $\operatorname{DCH}^{*}(A \times A)$ by O'Sullivan's Theorem 1.2 so that the cycles $p^{k, n}$ belong to $\mathrm{DCH}^{g}(A \times A)$; these are idempotents by O'Sullivan's Theorem.

Remark 2.7. The cycles $p^{k, n}$ can be defined explicitly in terms of the $\Lambda_{A}^{i}$ by carrying cohomological computations similar to [10, Proposition 1] or [44, Proposition 7.3] (note that in [44, Proposition 7.3] there is a sign error: $(-1)^{i}$ should read $\left.(-1)^{i+g}\right)$. Moreover, since the ChowKünneth projectors $\pi_{A}^{k}:=\sum_{n} p^{k, n}$ of Lemma 2.6 are symmetrically distinguished, they coincide with the ones of Deninger-Murre [17]. In particular, writing $\mathfrak{h}^{k}(A)$ for the direct summand of the Chow motive $\mathfrak{h}(A)$ corresponding to the Chow-Künneth projector $\pi_{A}^{k}$, we have the Beauville decomposition [11]:

$$
\mathrm{CH}^{i}(A)_{(j)}:=\mathrm{CH}^{i}\left(\mathfrak{h}^{i-2 j}(A)\right)=\left\{a \in \mathrm{CH}^{i}(A):[n]^{*} a=n^{i-2 j} a \text { for all } n \in \mathbb{Z}\right\} .
$$

Here, $[n]: A \rightarrow A$ is the multiplication-by- $n$ homomorphism.

Question 2.8 (generically defined cycles and symmetrically distinguished cycles). It is tempting to ask whether generically defined cycles on powers of abelian varieties are symmetrically distinguished in the sense of O'Sullivan [41], in particular whether generically defined cycles 
are invariant under the multiplication by -1 homomorphism. (All the explicit cycles that we consider that are generically defined are also symmetrically distinguished.) Since the Q-subalgebra of $\mathrm{CH}^{*}\left(A^{m}\right)$ consisting of symmetrically distinguished cycles injects in cohomology, and since Hodge classes on $A^{m}$ consist of polynomials in $p_{i}^{*} L$ and $p_{i, j}^{*} c_{1}\left(\mathcal{P}_{\mathcal{A}}\right)$ for $A$ very general (see Theorem 2.12), this would imply that generically defined cycles on $m$-fold powers of abelian varieties are polynomials in $p_{i}^{*} L$ and $p_{i, j}^{*} c_{1}\left(\mathcal{P}_{\mathcal{A}}\right)$; see also Proposition 3.11(a) below. This would constitute a generalization (with rational coefficients) of the Franchetta conjecture for abelian varieties; see the recent [19] where it is shown in particular that a generically defined cycle (with rational coefficients) of codimension 1 on polarized abelian varieties is a rational multiple of the polarization.

Given the fact that a general complex principally polarized abelian threefold is isomorphic to the Jacobian of a smooth projective curve of genus, one could be led to think that the Ceresa cycle (which for a very general such abelian threefold is not symmetrically distinguished) provides a generically defined cycle for principally polarized threefolds. This is however not the case. Indeed, the morphism $\mathcal{C}_{3, N} \rightarrow \mathcal{A}_{3, N}$ from the moduli space of genus 3 curves with level $N$ structure to the moduli space of principally polarized abelian threefolds with level $N$ structure $(N \geq 3)$ is a degree 2 morphism, due to the fact that a general curve of genus 3 has no non-trivial automorphism whereas an abelian variety always admits an involution. Since the Ceresa cycle is sent to minus itself under the multiplication by -1 homomorphism, we see that the Ceresa cycle is in fact fiberwise zero over $\mathcal{A}_{3, N}$. We refer to [40] for more details.

Question 2.9 (generically defined cycles on hyperKähler varieties). It is also tempting to ask whether the sub-ring of the Chow ring consisting of generically defined cycles on polarized hyperKähler varieties of a fixed deformation type injects into cohomology; see [21] for precise statements and some evidence. Note that contrary to the case of abelian varieties, we do not expect generically defined cycles to be sums of intersections of divisors or even Chern classes; for instance, for hyperKähler varieties that are deformations of $\operatorname{Hilb}^{n}(K 3)$, the Beauville-BogomolovFujiki class defines a generically defined Hodge class on the 2-fold product, and we expect the existence of a generically defined cycle $L$ in 2 -fold powers of such varieties whose cohomology class is the Beauville-Bogomolov-Fujiki class; see [44].

2.2. The generalized Hodge conjecture for very general abelian varieties. We recall the well-known fact that for a very general abelian variety the Hodge coniveau filtration coincides with the primitive filtration.

Definition 2.10. Let $(X, L)$ be a smooth projective complex variety of dimension $d$, equipped with a polarization $L$. The primitive filtration (with respect to $L$ ) is

$$
\mathrm{P}^{j} \mathrm{H}^{k}(X, \mathbb{Q})=\bigoplus_{r \geq j} L^{r} \mathrm{H}^{k-2 r}(X, \mathbb{Q})_{\text {prim }}
$$

where $\mathrm{H}^{i}(X, \mathbb{Q})_{\text {prim }}=\operatorname{ker}\left(L^{d-i+1}: \mathrm{H}^{i}(X, \mathbb{Q}) \rightarrow \mathrm{H}^{2 d-i+2}(X, \mathbb{Q})\right)$ for $i \leq d$, and is 0 for $i>d$.

Note that when $A$ is a very general abelian variety, there is up to scalar only one symmetric ample divisor on $A$. In particular, in this case, the primitive filtration does not depend on the choice of a polarization. The following theorem is folklore.

Theorem 2.11 (Generalized Hodge conjecture for very general abelian varieties). Let $A$ be a very general polarized complex abelian variety. Then

$$
\mathrm{P}^{*} \mathrm{H}^{k}(A, \mathbb{Q})=\mathrm{N}_{H}^{*} \mathrm{H}^{k}(A, \mathbb{Q})
$$

for all $k \geq 0$.

Proof. Since $A$ is very general, its Hodge group is dense in the symplectic group $\operatorname{Sp}\left(\mathrm{H}^{1}(A, \mathbb{Q})\right)$. The proof thus reduces to a representation-theoretic argument. We refer to Hain's argument in [18, Prop. 4.4], or to [26, p. 135]. 
2.3. The generalized Hodge conjecture for self-powers of very general abelian varieties. A crucial step towards the proof of Theorem 1 is the following generalization due to Hazama [26] of Theorem 2.11 to self-powers of $A$.

Theorem 2.12 (Hazama). Let $A$ be a very general polarized complex abelian variety. Then, denoting $\iota_{\Delta}: A^{m} \rightarrow A^{m} \times A^{m}$ the diagonal embedding, we have

$$
\mathrm{N}_{H}^{n} \mathrm{H}^{k}\left(A^{m}, \mathbb{Q}\right)=\sum_{Q}\left(\left(\iota_{\Delta}\right)_{*} Q\right)_{*} \mathrm{H}^{k-2 n}\left(A^{m}, \mathbb{Q}\right)
$$

for all $m, k \geq 0$, where the sum runs through all cycles $Q \in \mathrm{CH}^{n}\left(A^{m}\right)$ which are products of cycles of the form $\left(p_{i}\right)^{*} L,\left(p_{i, j}\right)^{*} P$. Here $p_{i}: A^{m} \rightarrow A$ and $p_{i, j}: A^{m} \rightarrow A^{2}$ are the natural projections, and $P \in \mathrm{CH}^{1}(A \times A)$ is the first Chern class of the Poincaré line-bundle (see $\left.§ 1.1\right)$.

Proof. This is due to Hazama [26, Th. 5.1]. (Note that a very general abelian variety is such that $\operatorname{End}^{0}(A)=\mathbb{Q}$ (hence of type I), and is such that its Hodge group coincides with its Lefschetz group (and hence stably nondegenerate in the terminology of [26])). The proof is representationtheoretic and involves understanding the irreducible representations of $\operatorname{Sp}\left(H^{1}(A, \mathbb{Q})\right)$ that appear as direct summands of the representations $\bigwedge^{k_{1}} H^{1}(A, \mathbb{Q}) \otimes \cdots \otimes \bigwedge^{k_{r}} H^{1}(A, \mathbb{Q})$ with $k_{1}+$ $\cdots+k_{r}=k$. For a proof, we also refer to Theorem 3.7, where we will generalize Hazama's theorem.

As a consequence, we can prove (a finer version of) Conjecture 1.6 for powers of a very general abelian variety:

Proposition 2.13. Let $A$ be a very general polarized complex abelian variety of dimension $g$, and let $m$ be an integer. Then for every integers $k$ and $n$ there exists an idempotent correspondence $q^{k, n} \in \mathrm{CH}^{g m}\left(A^{m} \times A^{m}\right)$ inducing the projection $\mathrm{H}^{*}\left(A^{m}, \mathbb{Q}\right) \rightarrow \mathrm{N}_{H}^{n} \mathrm{H}^{k}\left(A^{m}, \mathbb{Q}\right) \rightarrow \mathrm{H}^{*}\left(A^{m}, \mathbb{Q}\right)$, which is a linear combination of correspondences of the form

$$
\mathfrak{h}\left(A^{m}\right) \stackrel{\rho}{\longrightarrow} \mathfrak{h}\left(A^{m}\right)(n) \stackrel{\zeta}{\longrightarrow} \mathfrak{h}\left(A^{m}\right),
$$

where $\rho$ and $\zeta$ are both symmetrically distinguished cycles and generically defined cycles on $2 m$-fold powers of abelian varieties of dimension $g$. Moreover, such a correspondence is unique modulo homological equivalence.

Proof. By Theorem 2.12, we have

$$
\mathrm{N}_{H}^{n} \mathrm{H}^{k}\left(A^{m}, \mathbb{Q}\right)=\Gamma_{*} \mathrm{H}^{k-2 n}(B, \mathbb{Q}),
$$

where $B:=\coprod_{Q} A^{m}$ is the disjoint union of copies of $A^{m}$ indexed by the correspondences $Q$, and $\Gamma:=\sum_{Q}\left(\iota_{\Delta}\right)_{*} Q \in \mathrm{CH}^{g m+n}\left(B \times A^{m}\right)$. Since the correspondences $Q$ are symmetrically distinguished and generically defined for $2 m$-fold products of abelian varieties, the correspondence $\Gamma$ is symmetrically distinguished and generically defined for $2 m$-fold products of abelian varieties. We view $\Gamma$ as a morphism of Chow motives $\mathfrak{h}(B)(n) \rightarrow \mathfrak{h}\left(A^{m}\right)$. In the proof below, we are going to construct idempotent correspondences $q^{k, n}$, with the factorization property stated in the proposition, whose action on cohomology is the orthogonal projector on $\Gamma_{*} \mathrm{H}^{k-2 n}(B, \mathbb{Q})$, for all abelian varieties $A$ (the hypothesis that $A$ is very general is only used to compare $\Gamma_{*} \mathrm{H}^{k-2 n}(B, \mathbb{Q})$ with $\mathrm{N}_{H}^{n} \mathrm{H}^{k}\left(A^{m}, \mathbb{Q}\right)$; these coincide when $A$ is very general by Theorem 2.12).

By Lemma 2.6, the endomorphisms $p^{j, r} \in \operatorname{End}\left(\mathrm{H}^{*}\left(A^{\prime}, \mathbb{Q}\right)\right)$ are induced by cycles that belong to $\mathrm{DCH}^{m g}\left(A^{\prime} \times A^{\prime}\right)$ and are generically defined on 2 -fold products of abelian varieties $A^{\prime}$ of dimension $m g$. Restricting to $2 m$-fold products of abelian varieties of dimension $g$, we see by Remark 2.4 that the $p^{j, r} \in \operatorname{End}\left(\mathrm{H}^{*}\left(A^{m}, \mathbb{Q}\right)\right)$ are in fact induced by generically defined cycles on $2 m$-fold products of abelian varieties of dimension $g$. Hence, the endomorphisms $s_{j}:=$ $\sum_{r}(-1)^{r} p^{j, r} \in \operatorname{End}\left(\mathfrak{h}\left(A^{m}\right)\right)$ and the Chow-Künneth projectors $\pi^{j}:=\sum_{r} p^{j, r} \in \operatorname{End}\left(\mathfrak{h}\left(A^{m}\right)\right)$ are cycles that belong to $\mathrm{DCH}^{m g}\left(A^{m} \times A^{m}\right)$ and are generically defined on $2 m$-fold products of abelian varieties of dimension $g$. 
Denote $s:=\bigoplus_{Q} s_{k-2 n} \in \operatorname{End}(\mathfrak{h}(B))$ and $\Lambda_{B}=\coprod_{Q} \Lambda_{A^{m}}^{2 m g+2 n-k}$. Since the Hodge structure $\mathrm{H}^{2 g m-k+2 n}(B, \mathbb{Q})\left(=\bigoplus_{Q} \mathrm{H}^{2 g m-k+2 n}\left(A^{m}, \mathbb{Q}\right)\right)$, equipped with the pairing $\alpha \otimes \phi \mapsto \int_{B} \alpha \cup(s \circ$ $\left.\Lambda_{B}\right)_{*} \phi$, is polarized, we have (see e.g. [50, Lemma 1.6])

$$
\operatorname{im}\left(\left(\Gamma \circ s \circ \Lambda_{B} \circ{ }^{t} \Gamma \circ \Lambda_{A^{m}}^{k}\right)_{*}\right)=\operatorname{im}\left(\Gamma_{*}\right)=\mathrm{N}_{H}^{n} \mathrm{H}^{k}\left(A^{m}, \mathbb{Q}\right) .
$$

Moreover the correspondence $s \circ \Lambda_{B} \circ{ }^{t} \Gamma \circ \Lambda_{A^{m}}^{k}$ acts as zero on the orthogonal complement of $\Gamma_{*} \mathrm{H}^{k-2 n}(B, \mathbb{Q})$. By the theorem of Cayley-Hamilton, we may thus express the orthogonal projector on $\Gamma_{*} \mathrm{H}^{k-2 n}(B, \mathbb{Q})$ as a polynomial (with zero constant term) in the endomorphism $\left(\Gamma \circ s \circ \Lambda_{B} \circ{ }^{t} \Gamma \circ \Lambda_{A^{m}}^{k}\right)_{*} \in \operatorname{End}\left(\mathrm{H}\left(A^{m}, \mathbb{Q}\right)\right)$. This shows that the orthogonal projector on $\Gamma_{*} \mathrm{H}^{k-2 n}(B, \mathbb{Q})$ is induced by a cycle that is a linear combination of cycles with the factorization property stated in the proposition.

Finally, concerning the uniqueness of $q^{k, n}$ modulo homological equivalence, let us prove more generally that an endomorphism of a Hodge structure $H$ of weight $k$, with image $\mathrm{N}_{H}^{n} H$ is unique. Assume $q$ and $q^{\prime}$ are two such endomorphisms. By definition of the Hodge coniveau filtration, $q$ and $\operatorname{id}_{H}-q^{\prime}$ are mutually orthogonal projectors. Therefore $q$ and $q^{\prime}$ commute; we conclude by using the elementary fact that two idempotent endomorphisms of a vector space coincide when they commute with one another and have the same image.

Remark 2.14 (Refined Chow-Künneth decompositions). Without going into the details, we simply note that Proposition 2.13 shows that the refined Chow-Künneth projectors of [50] can be constructed unconditionally for the powers of a very general abelian variety. Due to Proposition 3.12 and Corollary 3.13 below, the same holds for self-powers of elliptic curves or abelian surfaces. In particular, since generalized Kummer varieties are motivated by an abelian surface (see §4.4), they admit a refined Chow-Künneth decomposition in the sense of [50].

2.4. Generically defined cycles and the generalized Bloch conjecture. Our main result concerning generically defined cycles is the following slight generalization of Theorem 1 :

Theorem 2.15. Let $\gamma \in \mathrm{CH}^{*}\left(A^{l} \times A^{m}\right)$ be a generically defined cycle on the $(l+m)$-fold power of a polarized complex abelian variety $A$ of dimension $g$. Assume that $\gamma^{*} \mathrm{H}^{*}\left(A^{m}, \mathbb{Q}\right) \subseteq \mathrm{N}_{H}^{n} \mathrm{H}^{*}\left(A^{l}, \mathbb{Q}\right)$ for some (equivalently, for all) polarized complex abelian variety $A$ of dimension $g$ and degree $d^{2}$. We have:

(1) If $l=m$ and $\gamma \in \mathrm{CH}^{m g}\left(A^{m} \times A^{m}\right)$, then $\gamma_{*}$ acts nilpotently on $\mathrm{CH}_{r}\left(A^{m}\right)$ for all $r<n$. In particular, if $\gamma$ is an idempotent correspondence, then $\gamma_{*} \mathrm{CH}_{r}\left(A^{m}\right)=0$ for all $r<n$.

(2) If $\gamma$ is a symmetrically distinguished correspondence, then $\gamma_{*} \mathrm{CH}_{r}\left(A^{m}\right)=0$ for all $r<n$.

Proof. The notations are those of Proposition 2.13 and its proof. First assume that $A$ is very general. By assumption, ${ }^{t} \gamma$ has same homology class as $\sum_{k} q^{k, n} \circ{ }^{t} \gamma$. In particular, after transposing the above equality, $\gamma$ is a linear combination of morphisms that factor through the morphism of homological motives ${ }^{t} \Gamma: \mathfrak{h}^{\text {hom }}\left(A^{m}\right) \rightarrow \mathfrak{h}^{\text {hom }}(B)(-n)=\bigoplus_{Q} \mathfrak{h}^{\text {hom }}\left(A^{m}\right)(-n)$. Since all the cycles considered in the proof of Proposition 2.13 are generically defined for $2 m$-fold powers of abelian varieties of dimension $g$, the above conclusion in fact holds without assuming that the abelian variety $A$ is very general.

(1) We are assuming that $\gamma$ is a self-correspondence on $A^{m}$ of degree 0 ; i.e., that it is a morphism $\mathfrak{h}\left(A^{m}\right) \rightarrow \mathfrak{h}\left(A^{m}\right)$. By finite-dimensionality of the motive of abelian varieties [29], some power of $\gamma$, say $\gamma^{\circ N}$, factors through the morphism of Chow motives ${ }^{t} \Gamma: \mathfrak{h}\left(A^{m}\right) \rightarrow \mathfrak{h}(B)(-n)$. Therefore the action of $\gamma^{\circ N}$ on $\mathrm{CH}_{r}\left(A^{m}\right)$ factors through $\mathrm{CH}_{r-n}(B)$; the group $\mathrm{CH}_{r-n}(B)$ is obviously zero for $r<n$.

(2) Finally, in order to see that $\gamma_{*} \mathrm{CH}_{r}\left(A^{m}\right)=0$ for all $r<n$ if $\gamma$ is assumed to be symmetrically distinguished, it suffices to note that all the cycles appearing in Proposition 2.13 and its proof are symmetrically distinguished, so that ${ }^{t} \gamma$ is equal to $\sum_{k} q^{k, n} \circ{ }^{t} \gamma$ modulo rational equivalence, and hence is a linear combination of morphisms that factor through the morphism of motives ${ }^{t} \Gamma: \mathfrak{h}\left(A^{m}\right) \rightarrow \mathfrak{h}(B)(-n)=\bigoplus_{Q} \mathfrak{h}\left(A^{m}\right)(-n)$. 


\section{LEFSCHETZ REPRESENTATIONS}

3.1. The Lefschetz group. In this paragraph, we fix definitions and notations as well as recall the basic properties of the Lefschetz group. A rational Hodge structure $H$ of pure weight $k$ can be described as a $\mathbb{Q}$-vector space of finite dimension with a homomorphism of $\mathbb{R}$-groups $\operatorname{Res}_{\mathbb{C} / \mathbb{R}} \mathbb{G}_{m} \rightarrow \mathrm{GL}\left(H_{\mathbb{R}}\right)$, where Res denotes restriction of scalars à la Weil. The Mumford-Tate group $\mathrm{MT}(H)$ of a rational Hodge structure $H$ is the Q-Zariski closure of the image of this homomorphism; it is connected. If $H$ is of pure weight, we will be interested in a smaller group called the Hodge group of $H: \operatorname{Hdg}(H)$ is the Q-Zariski closure of the image of the circle group $\operatorname{Ker}\left(\operatorname{Res}_{\mathbb{C} / \mathbb{R}} \mathbb{G}_{m} \rightarrow \mathbb{G}_{m}\right) \rightarrow \mathrm{GL}\left(H_{\mathbb{R}}\right)$. (Concretely, a Hodge structure $H$ of weight $k$ determines a homomorphism $\lambda_{H}: S^{1} \rightarrow \mathrm{GL}\left(H_{\mathbb{R}}\right)$ where $S^{1}$ is the unit circle in the complex plane, such that $\lambda_{H}(z)$ acts on $H^{p, q}$ by multiplication by $z^{p-q}$, and conversely such a homomorphism induces an eigenspace decomposition of $H_{\mathbb{C}}$ that satisfies the Hodge symmetry $\left.\overline{H^{p, q}}=H^{q, p}\right)$. The Hodge group is a connected group characterized by the property that its invariants in $H^{m} \otimes\left(H^{\vee}\right)^{n}$ are precisely the Hodge classes for all non-negative integers $m$ and $n$. Moreover the Hodge substructures of $H^{m} \otimes\left(H^{\vee}\right)^{n}$ are precisely the sub-representations of the Hodge group of $H$. The element $C:=\lambda_{H}(i)$ is called the Weil operator. A polarization of $H$ is a morphism of Hodge structures $\phi: H \otimes_{\mathbb{Q}} H \rightarrow \mathbb{Q}(-k)$ such that $\phi(x, C y)$ is symmetric and positive definite on $H_{\mathbb{R}}$. When $H$ admits a polarization, then its Mumford-Tate and Hodge groups are reductive.

Let $A$ be a complex abelian variety. Cup-product defines an isomorphism of graded $\mathbb{Q}$-algebras $\bigwedge^{*} \mathrm{H}^{1}(A, \mathbb{Q}) \rightarrow \mathrm{H}^{*}(A)$. Via the isomorphism

$$
\mathrm{H}^{2}(A, \mathbb{Q}) \simeq \operatorname{Hom}\left(\bigwedge^{2} V(A), \mathbb{Q}(1)\right)
$$

the cohomology class of a divisor $D$ on $A$ defines a skew-symmetric pairing $\phi_{D}: V(A) \times$ $V(A) \rightarrow \mathbb{Q}(1)$, where $V(A):=\mathrm{H}_{1}(A, \mathbb{Q})$. When $D$ is ample, $\phi_{D}$ is non-degenerate and defines a polarization on the $\mathbb{Q}$-Hodge structure $V(A)$. We let $\rho_{D}$ denote the involution of the $\mathbb{Q}$-algebra $\operatorname{End}_{\mathbb{Q}}(V(A))$, which to an endomorphism of $V(A)$ associates its adjoint with respect to $\phi_{D}$; its restriction to

$$
\operatorname{End}^{0}(A):=\operatorname{End}(A) \otimes_{\mathbb{Z}} \mathbb{Q}
$$

is the Rosati involution defined by $D$. By definition, the Hodge group $\operatorname{Hdg}(A)$ of a complex abelian variety $A$ is the Hodge group attached to the polarized $\mathbb{Q}$-Hodge structure

$$
V(A):=\mathrm{H}_{1}(A, \mathbb{Q}) .
$$

Due to the semi-simplicity of the category of polarized Q-Hodge structures, the Mumford-Tate group and the Hodge group of a polarized Hodge structure are reductive groups.

Definition 3.1. For a complex abelian variety $A$ endowed with a polarization $L$, the Lefschetz group $L(A)$ is defined to be the algebraic subgroup of $\mathrm{GL}(V(A))$ such that, for all commutative Q-algebras $R$,

$$
L(A)(R)=\left\{\gamma \in C(A) \otimes_{\mathbb{Q}} R: \rho_{L}(\gamma) \gamma=1\right\} .
$$

Here $C(A)$ is the centralizer of $\operatorname{End}^{0}(A)$ in $\operatorname{End}_{\mathbb{Q}}(V(A))$. The Lefschetz group can also be viewed as the centralizer of $\operatorname{End}^{0}(A)$ in $\operatorname{Sp}\left(V(A), \phi_{L}\right)$.

The Lefschetz group does not depend of the choice of a polarization: given any two ample line-bundles $\mathcal{L}$ and $\mathcal{L}^{\prime}$, there is an element $\eta \in \operatorname{End}^{0}(A)$ and a positive integer $m$ such that $m \phi_{\mathcal{L}}=\phi_{\mathcal{L}^{\prime}} \eta$. In what follows, the polarization will usually be understood from the context, and we will therefore write simply $\rho$ for the Rosati involution, and $\phi$ for the skew-symmetric form. In general, we have the inclusions

$$
\operatorname{Hdg}(A) \subseteq L(A) \subseteq \operatorname{Sp}(V(A), \phi) .
$$

The Lefschetz group of $A$ naturally acts on the Q-vector spaces $V(A)^{\otimes n} \otimes\left(V(A)^{\vee}\right)^{\otimes m}$, and we will refer to these as Lefschetz representations. While the Hodge group doesn't behave well with respect to products, the Lefschetz group enjoys the following property: 
Lemma 3.2 (Murty [39]). If $A$ is isogenous to a product $A_{1}^{m_{1}} \times \cdots \times A_{s}^{m_{s}}$, with the $A_{i}$ simple and pairwise non-isogenous, then

$$
L(A) \cong L\left(A_{1}\right) \times \cdots \times L\left(A_{s}\right)
$$

with the factor $L\left(A_{i}\right)$ acting diagonally on $H_{1}\left(A_{i}, \mathbb{Q}\right)^{\oplus m_{i}}$ and acting as zero on $H_{1}\left(A_{j}, \mathbb{Q}\right)^{\oplus m_{j}}$ for $j \neq i$.

Recall that $\mathrm{R}^{*}(A) \subset \mathrm{CH}^{*}(A)$ denotes the $\mathbb{Q}$-sub-algebra generated by symmetric divisors and that it maps isomorphically onto its image $\overline{\mathrm{R}}^{*}(A)$ in $\mathrm{H}^{*}(A, \mathbb{Q})$ via the cycle class map by O'Sullivan's Theorem 1.2. The statement of the following theorem is taken from Milne [35, Thm. 3.2] where it is proved more generally for abelian varieties defined over any algebraically closed fields, but its origin can be traced back to work of Tankeev [48], Ribet [43], Murty [39], and Hazama [25].

Theorem 3.3. The Lefschetz group $L(A)$ of a complex abelian variety $A$ is such that $\overline{\mathrm{R}}^{s}\left(A^{r}\right)=$ $\mathrm{H}^{2 s}\left(A^{r}, \mathbb{Q}\right)^{L(A)}$ inside $\mathrm{H}^{2 s}\left(A^{r}, \mathbb{Q}\right)$ for all non-negative integers $r$ and $s$.

Since the Hodge classes in $\mathrm{H}^{2 s}\left(A^{r}, \mathbb{Q}\right)$ are precisely the invariant classes under the action of the Hodge group $\operatorname{Hdg}(A)$, it follows from the Lefschetz $(1,1)$-theorem that the Hodge conjecture holds for powers of abelian varieties for which the inclusion $\operatorname{Hdg}(A) \subseteq L(A)$ is an equality. This is for example the case for elliptic curves, and abelian varieties of prime dimension; see [48] and [43].

3.2. Lefschetz groups and the Albert classification. Let $A$ be a complex abelian variety. The proof of Theorem 3.3 proceeds through the computation of the Lefschetz group $L(A)$. We start this paragraph by reviewing how the Lefschetz group of a simple complex abelian variety can be computed via the characterization of the possible algebras $\operatorname{End}^{0}(A)$; for this we follow Murty [39], and we refer to Shimura [46] for the classification of such algebras via the Albert classification of division algebras with a positive involution. Set $D:=\operatorname{End}^{0}(A)=\operatorname{End}(A) \otimes_{\mathbb{Z}} \mathbb{Q}$. The Rosati involution $\rho$ of the semi-simple $\mathbb{Q}$-algebra $D$ induced by a polarization of $A$ defines a positive involution of $D$ in the sense that $D$ has finite dimension over $\mathbb{Q}$ and the reduced trace $\operatorname{tr}_{D / \mathbb{Q}}(x \rho(x))$ is positive for all non-zero $x \in D$. From now on, we assume that $A$ is simple; in that case, $D$ is a division algebra. The involution $\rho$ restricts to a positive involution of the center $Z$ of $D$, and we denote $F$ the set of elements $z \in Z$ such that $\rho(z)=z$. As we have $\operatorname{tr}\left(z^{2}\right)>0$ for every non-zero element $z$ of $F$, the field $F$ must be a totally real field. We set $d:=[D: Z]^{1 / 2}$ and $f:=[F: \mathbb{Q}]$. According to Albert the following possibilities can occur for division algebras endowed with a positive involution:

Type I. $D=F$ is a totally real field;

Type II. $D$ is a central division algebra over $F$ such that $D \otimes_{\mathbb{Q}} \mathbb{R}$ is isomorphic to the product of $f$ copies of the matrix algebra $\mathrm{M}_{2}(\mathbb{R})$;

Type III. $D$ is a central division algebra over $F$ such that $D \otimes_{\mathbb{Q}} \mathbb{R}$ is isomorphic to the product of $f$ copies of the quaternion algebra $\mathbb{H}$;

Type IV. $D$ is a central division algebra over a totally imaginary quadratic extension $F_{0}$ of $F$.

Accordingly a simple abelian variety $A$ is said to have type I, II, III, or IV, if $D=\operatorname{End}^{0}(A)$ has type I, II, III, or IV, respectively. For endomorphism rings of simple complex abelian varieties of dimension $g$, there are further dimension restrictions on the division algebras, coming from that fact that $D$ acts faithfully on the $2 g$-dimensional vector space $V(A)=\mathrm{H}_{1}(A, \mathbb{Q})$ (and the fact that the action of $D$ commutes with the complex structure for type I), namely $f \mid g$ for type I, $2 f \mid g$ for types II and III, and $f d^{2} \mid 2 g$ for type IV. Shimura [46] showed that every division algebra with a positive involution occurs as the endomorphism algebra of a simple complex abelian variety, except in 5 exceptional cases; in particular [46, Prop. 15], for a simple abelian variety of type III, $2 f$ must divide $g$ strictly. 
We now fix a skew-symmetric non-degenerate pairing $\phi: V(A) \times V(A) \rightarrow \mathbb{Q}$ determined by a polarization of $A$, via (3). For each type, there exist a unique non-degenerate $F$-bilinear form ${ }^{2}$

$$
B: V(A) \times V(A) \rightarrow D
$$

such that $\phi(x, y)=\operatorname{tr}_{D / \mathbb{Q}} B(x, y), B(a x, b y)=a B(x, y) b$, and $B(y, x)=-\rho \circ B(x, y)$ for all $x, y \in V(A)$ and all $a, b \in D$. The Lefschetz group is then the restriction of scalars, from $F$ to $\mathbb{Q}$, of the unitary group of $B$ :

$$
L(A)=\operatorname{Res}_{F / \mathrm{Q}} \mathrm{U}(B)=\operatorname{Res}_{F / \mathrm{Q}} \operatorname{Aut}_{D}(V(A), B) .
$$

Let $S$ be the set of embeddings of $F$ into $\mathbb{R}$. We can then write

$$
V(A)_{\mathbb{R}}=\bigoplus_{\lambda \in S} V_{\lambda},
$$

where $V_{\lambda}:=V(A) \otimes_{F, \lambda} \mathbb{R}=\left\{v \in V(A)_{\mathbb{R}}: f(v)=\lambda(f) v\right.$ for all $\left.f \in F\right\}$ is a real vector space of dimension $2 g / f$. In fact, since $D$ commutes with $\operatorname{Hdg}(A)_{\mathbb{R}}, V_{\lambda}$ is a real Hodge sub-structure of $V(A)_{\mathbb{R}}$. Since $L(A)$ commutes with the action of $F$, we have $L(A)_{\mathbb{R}} \subseteq \prod \mathrm{GL}\left(V_{\lambda}\right)$ and thus

$$
L(A)_{\mathbb{R}}=\prod_{\lambda \in S} L_{\lambda}, \quad \text { with } L_{\lambda}=\operatorname{Aut}_{D_{\lambda}}\left(V_{\lambda}, B_{\lambda}\right),
$$

where $L_{\lambda}$ acts trivially on $V_{\lambda^{\prime}}$ unless $\lambda=\lambda^{\prime}$. Here $D_{\lambda}:=D \otimes_{F, \lambda} \mathbb{R}$ and $B_{\lambda}$ is the non-degenerate real bilinear form that is the restriction of $B \otimes_{\mathbb{Q}} \mathbb{R}$ to $V_{\lambda} \times V_{\lambda}$.

For types II and III, there exists an $F$-basis $1, \alpha, \beta, \alpha \beta$ for $D$, with $\alpha^{2}$ totally negative, $\beta^{2}$ totally positive for type II and totally negative for type III, and $\alpha \beta=-\beta \alpha$. Denoting $E:=F[\alpha]$, we have $D=E \oplus E \beta$, and we can write

$$
B(x, y)=B_{1}(x, y)+B_{2}(x, y) \beta, \quad \text { with } B_{1}(x, y), B_{2}(x, y) \in E .
$$

Then $B_{1}: V(A) \times V(A) \rightarrow E$ is a non-degenerate skew-Hermitian form, and $B_{2}: V(A) \times V(A) \rightarrow$ $E$ is a non-degenerate skew-symmetric form for type II and a non-degenerate symmetric form for type III. Given an embedding $\lambda: F \hookrightarrow \mathbb{R}$, we denote $\sigma, \bar{\sigma}: E \hookrightarrow \mathbb{C}$ the conjugate extensions of $\lambda$ to $E$. We define $V_{\sigma}:=V \otimes_{E, \sigma} \mathbb{C}$, and we remark that $B_{1, \sigma}:=\left.\left(B_{1} \otimes_{\mathbb{Q}} \mathbb{C}\right)\right|_{V_{\sigma} \times V_{\sigma}}=0$, while $B_{2, \sigma}:=\left.\left(B_{2} \otimes_{\mathbb{Q}} \mathbb{C}\right)\right|_{V_{\sigma} \times V_{\sigma}}$ is non-degenerate (and similarly with $\bar{\sigma}$ in place of $\sigma$ ).

The group $L_{\lambda}$ and its action on $V_{\lambda}$ are given as follows (see [39], but also [35]):

Type I. $L_{\lambda}=\operatorname{Sp}_{\frac{2 g}{f}}\left(V_{\lambda}, B_{\lambda}\right)$ is a symplectic group acting via its standard representation on $V_{\lambda}$

Type II. $L_{\lambda} \otimes_{\mathbb{R}} \mathbb{C}=\operatorname{Sp}_{\frac{g}{f}}\left(V_{\sigma}, B_{2, \sigma}\right)$ is a symplectic group acting on $V_{\lambda} \otimes_{\mathbb{R}} \mathbb{C}=V_{\sigma} \oplus V_{\bar{\sigma}}$ as one copy of the standard representation and one copy of its contragredient representation (which is isomorphic to the standard representation).

Type III. $L_{\lambda} \otimes_{\mathbb{R}} \mathbb{C}=\mathrm{O}_{\frac{g}{f}}\left(V_{\sigma}, B_{2, \sigma}\right)$ is an orthogonal group acting on $V_{\lambda} \otimes_{\mathbb{R}} \mathbb{C}=V_{\sigma} \oplus V_{\bar{\sigma}}$ as one copy of the standard representation and one copy of its contragredient representation (which is isomorphic to the standard representation). ${ }^{3}$

Type IV. $L_{\lambda} \otimes_{\mathbb{R}} \mathbb{C}=\mathrm{GL}_{\frac{g}{d f}}(\mathbb{C})$ acts on $V_{\lambda} \otimes_{\mathbb{R}} \mathbb{C}$ as the direct sum of the standard representation and its contragredient representation.

In particular, the Lefschetz group $L(A)$ is a reductive group. The Lefschetz group $L(A)$ was first computed by Ribet [43] for type I and IV in the case $D=F$. It was computed in general by Murty $[39]^{4}$.

Here is a useful basic fact (which is made more precise in the proof of [6, Théorème 6.1]) that can be derived from the reductiveness of the Lefschetz group:

\footnotetext{
${ }^{2}$ The trace pairing $D \times D \rightarrow \mathbb{Q},(a, b) \mapsto \operatorname{tr}_{D / \mathbb{Q}}(a b)$ is non-degenerate, and $B(x, y)$ is the unique element in $D$ satisfying $\operatorname{tr}_{D / \mathbb{Q}}(a B(x, y))=\phi(a x, y)$ for all $a \in D$.

${ }^{3}$ In that case $\frac{g}{f}$ is an even number $\geq 4$ by [46, Prop. 15].

${ }^{4}$ For type III Murty finds that $L_{\lambda} \otimes_{\mathbb{R}} \mathbb{C}$ is a special orthogonal group; this is because, contrary to the convention we adopted here, he considers the connected component of the identity of the Lefschetz group.
} 
Lemma 3.4. Let $A$ be a complex abelian variety, and let $H \subseteq \mathrm{H}^{*}(A, \mathbb{Q})$ be a Hodge substructure. Then $H \subseteq \mathrm{H}^{*}(A, \mathbb{Q})$ is a $L(A)$-sub-representation if and only if there exists a projector $\mathrm{H}^{*}(A, \mathbb{Q}) \rightarrow \mathrm{H}^{*}(A, \mathbb{Q})$ with image $H$ that is induced by an idempotent correspondence in $\mathrm{R}^{\operatorname{dim} A}(A \times A)$.

Proof. Thanks to the fact that $L(A)$ is reductive, $H$ is a $L(A)$-sub-representation of $\mathrm{H}^{*}(A, \mathbb{Q})$ if and only if there exists a $L(A)$-invariant projector $\mathrm{H}^{*}(A, \mathbb{Q}) \rightarrow \mathrm{H}^{*}(A, \mathbb{Q})$ with image $H$. By Theorem 3.3 a $L(A)$-invariant projector $\mathrm{H}^{*}(A, \mathbb{Q}) \rightarrow \mathrm{H}^{*}(A, \mathbb{Q})$ with image $H$ is induced by an idempotent correspondence in $\overline{\mathrm{R}}^{\operatorname{dim} A}(A \times A)$, and by O'Sullivan's theorem 1.2 such an idempotent correspondence can be lifted to an idempotent correspondence in $\mathrm{R}^{\operatorname{dim} A}(A \times A)$.

Finally, we observe that, since $D \otimes_{\mathbb{Q}} \mathbb{C} \cong \operatorname{End}_{\operatorname{Hdg}(A)_{\mathbb{C}}}\left(\mathrm{H}_{1}(A, \mathbb{C})\right)$, the action of $D \otimes_{\mathbb{Q}} \mathbb{C}$ on $V(A)_{\mathbb{C}}=H_{1}(A, \mathbb{C})$ commutes with the Hodge decomposition. In particular, if $E$ is a field sitting inside $D$, the decomposition $V_{\mathbb{C}}=\oplus_{\sigma: E \hookrightarrow \mathbb{C}} V_{\sigma}$ is compatible with the Hodge decomposition, so that writing $V_{\sigma}^{1,0}=V^{1,0} \cap V_{\sigma}$ and similarly for $V_{\sigma}^{0,1}$, we have

$$
V_{\sigma}=V_{\sigma}^{1,0} \oplus V_{\sigma}^{0,1} \text {. }
$$

We note also that

$$
\overline{V_{\sigma}^{1,0}}=V_{\bar{\sigma}}^{0,1} .
$$

The following lemma will be crucial to the proof of Theorem 3.7. Since it does not hold in general for simple abelian varieties of type IV (e.g. CM elliptic curves), our focus until §3.5 will be on abelian varieties of totally real type.

Lemma 3.5. Let $A$ be a simple complex abelian variety of type I, II or III. Let $E$ be a maximal subfield of $\operatorname{End}^{0}(A)$, which we choose as above to be a CM field for types II and III. Let $V_{\sigma}:=$ $V(A) \otimes_{E, \sigma} \mathbb{C}$ for an embedding $\sigma: E \hookrightarrow \mathbb{C}$. Then the decomposition (4) is a decomposition into isotropic subspaces for the non-degenerate form ${ }^{5}$

$$
B_{\sigma}:=\left.B_{\mathbb{C}}\right|_{V_{\sigma} \times V_{\sigma}}: V_{\sigma} \times V_{\sigma} \rightarrow D \otimes_{\mathbb{Q}} \mathbb{C} .
$$

In particular, $V_{\sigma}$ is "numerically Hodge symmetric", meaning that

$$
\operatorname{dim}_{\mathbb{C}} V_{\sigma}^{1,0}=\operatorname{dim}_{\mathbb{C}} V_{\sigma}^{0,1} .
$$

Proof. Recall that, for $x, y \in V_{\mathbb{C}}, B_{\mathbb{C}}(x, y)$ is the unique element in $D \otimes_{\mathbb{Q}} \mathbb{C}$ such that

$$
\operatorname{tr}_{D \otimes_{\mathbb{Q}} \mathbb{C} / \mathbb{C}}\left(a B_{\mathbb{C}}(x, y)\right)=\phi_{\mathbb{C}}(a x, y), \quad \text { for all } a \in D \otimes_{\mathbb{Q}} \mathbb{C} .
$$

Since $V^{1,0}$ and $V^{0,1}$ are isotropic subspaces for the form $\phi_{\mathbb{C}}$ and since the action of $D \otimes_{\mathbb{Q}} \mathbb{C}$ on $V_{\mathbb{C}}$ is compatible with the Hodge decomposition, we deduce that $B_{\mathbb{C}}(x, y)=0$ for all $x, y \in V^{1,0}$ (resp. for all $\left.x, y \in V^{0,1}\right)$. The lemma follows by restricting to the $\sigma$-component in the decomposition $V_{\mathbb{C}}=\oplus_{\sigma: E \hookrightarrow \mathbb{C}} V_{\sigma}$.

In summary, for simple abelian varieties of totally real type, Lemma 3.5 provides the following relations between the Hodge decomposition and the decomposition of the Lefschetz group after base-change :

Type I. The Hodge decomposition $V_{\lambda} \otimes_{\mathbb{R}} \mathbb{C}=V_{\lambda}^{1,0} \oplus V_{\lambda}^{0,1}$ is a decomposition into isotropic subspaces for the non-degenerate skew-symmetric form $\phi_{\lambda} \otimes_{\mathbb{R}} \mathbb{C}$;

Type II. The Hodge decomposition $V_{\sigma}=V_{\sigma}^{1,0} \oplus V_{\sigma}^{0,1}$ is a decomposition into isotropic subspaces for the non-degenerate skew-symmetric form $B_{2, \sigma}$;

Type III. The Hodge decomposition $V_{\sigma}=V_{\sigma}^{1,0} \oplus V_{\sigma}^{0,1}$ is a decomposition into isotropic subspaces for the non-degenerate symmetric form $B_{2, \sigma}$.

\footnotetext{
${ }^{5}$ Note that for types II and III, $B_{\sigma}=B_{2, \sigma}$.
} 
3.3. Around Weyl's construction. Let $V$ denote the standard representation of one of the classical groups $\mathrm{Sp}_{2 n}$ or $\mathrm{O}_{2 n}$. Precisely, given a basis $\left(e_{1}, \ldots, e_{n}, e_{-1}, \ldots, e_{-n}\right)$ of $V$, we will be interested in the representations of the following groups:

(a) $G=\operatorname{Sp}_{2 n}(V, Q)$, where $Q$ is the skew-symmetric bilinear form dual to

$$
\psi=\sum_{i=1}^{n} e_{i} \otimes e_{-i}-e_{-i} \otimes e_{i} \in V \otimes V .
$$

(b) $G=\mathrm{O}_{2 n}(V, Q)$, where $Q$ is the symmetric bilinear form dual to

$$
\psi=\sum_{i=1}^{n} e_{i} \otimes e_{-i}+e_{-i} \otimes e_{i} \in V \otimes V .
$$

For each pair $I=\{p<q\}$ of integers between 1 and $d$, the skew-symmetric form $Q$ (resp. the symmetric form $Q$ ) determines a contraction

$$
\begin{gathered}
\Phi_{I}: V^{\otimes d} \rightarrow V^{\otimes(d-2)}, \\
v_{1} \otimes \cdots \otimes v_{d} \mapsto Q\left(v_{p}, v_{q}\right) v_{1} \otimes \cdots \otimes \hat{v}_{p} \otimes \cdots \otimes \hat{v}_{q} \otimes \cdots \otimes v_{d},
\end{gathered}
$$

where a 'hat' means that the term is omitted. Denote $V^{\langle d\rangle}$ the intersection of the kernels of all these contractions, i.e.,

$$
V^{\langle d\rangle}:=\bigcap_{I} \operatorname{ker}\left(\Phi_{I}\right)
$$

We can also define

$$
\Psi_{I}: V^{\otimes(d-2)} \rightarrow V^{\otimes d}
$$

by inserting $\psi$ in the $p, q$ factors. We have a direct sum of $G$-representations (see [24, Ex. 17.13] for the case $G=\mathrm{Sp}_{2 n}$; the case $G=\mathrm{O}_{2 n}$ is similar)

$$
V^{\otimes d}=V^{\langle d\rangle} \oplus \sum_{I} \operatorname{im}\left(\Psi_{I}\right)
$$

By considering the action of the symmetric group $\mathfrak{S}_{d}$ on $V^{\otimes d}$, we also have a direct sum decomposition of $\mathfrak{S}_{d}$-representations [24, Ex. 4.50]

$$
V^{\otimes d}=\bigoplus_{\lambda \dashv d} \mathbb{S}_{\lambda} V
$$

Here the direct sum runs through all standard Young tableaux in $d$ entries, and $\mathbb{S}_{\lambda} V$ is the Schur symmetrizer attached to the underlying Young diagram. Moreover, for each standard Young tableau $\lambda$, there is an idempotent $p_{\lambda} \in \mathbb{Q}\left[\mathfrak{S}_{d}\right]$ (a rational multiple of the Young symmetrizer $c_{\lambda}$ ) such that $\mathbb{S}_{\lambda} V=p_{\lambda} \cdot V^{\otimes d}$, and these idempotents are mutually orthogonal meaning that $p_{\lambda} p_{\mu}=0$ for two distinct standard Young tableaux $\lambda$ and $\mu$.

Clearly a permutation $\sigma \in \mathfrak{S}_{d}$ commutes with the decomposition (5), and hence so do the idempotents $p_{\lambda}$. It follows that $V^{\langle d\rangle}$ further decomposes into a direct sum of $G$-representations as

$$
V^{\langle d\rangle}=\bigoplus_{\lambda \dashv d} \mathbb{S}_{\langle\lambda\rangle} V, \quad \text { where } \mathbb{S}_{\langle\lambda\rangle} V:=\mathbb{S}_{\lambda} V \cap V^{\langle d\rangle} .
$$

In order to state the next proposition, we need to introduce some notations. We follow Bourbaki [15, Chap. VIII, $\S 13.3 \& \S 13.4]$. Let $E_{i, j}$ be the $2 n \times 2 n$ matrix expressed in the basis $\left(e_{1}, \ldots, e_{n}, e_{-1}, \ldots, e_{-n}\right)$ whose entries are all zero except for the $(i, j)$-th entry which is 1 . For $1 \leq i \leq n$, we define

$$
H_{i}:=E_{i, i}-E_{-i,-i}
$$

and we let $\left(\varepsilon_{1}, \ldots, \varepsilon_{n}\right)$ be the dual basis of $\left(H_{1}, \ldots, H_{n}\right)$.

Given a standard Young tableau $\lambda$ on $d$ entries, we denote $\left(\lambda_{1} \geq \lambda_{2} \geq \cdots \geq \lambda_{d}\right)$ the underlying partition of $d$. Subsequently, the number $d=\sum_{i} \lambda_{i}$ will also be referred to as the length of $\lambda$ and will be denoted $\ell(\lambda)$. 
Proposition 3.6. Assume that $G$ is either $\mathrm{Sp}_{2 n}$, or $\mathrm{O}_{2 n}$ with $n>1$. Then $\mathbb{S}_{\langle\lambda\rangle} V$ is an irreducible representation of $G$.

(a) If $G=\mathrm{Sp}_{2 n}$, then $\mathbb{S}_{\langle\lambda\rangle} V \neq 0$ if and only if $\lambda_{n+1}=0$; in that case $\mathbb{S}_{\langle\lambda\rangle} V$ is the irreducible representation of $\mathfrak{s p}_{2 n}$ with highest weight $\lambda_{1} \varepsilon_{1}+\cdots+\lambda_{n} \varepsilon_{n}$.

(b) If $G=\mathrm{O}_{2 n}$, then $\mathbb{S}_{\langle\lambda\rangle} V \neq 0$ if and only if the sum of the lengths of the first two columns of the Young tableau $\lambda$ is at most $2 n{ }^{6}$

- If $\lambda=\left(\lambda_{1} \geq \cdots \geq \lambda_{n}=0\right)$, then $\mathbb{S}_{\langle\lambda\rangle} V$ is the irreducible representation of $\mathfrak{s o}_{2 n}$ with highest weight $\lambda_{1} \varepsilon_{1}+\cdots+\lambda_{n} \varepsilon_{n}$.

- If $\lambda=\left(\lambda_{1} \geq \cdots \geq \lambda_{n}>0\right)$, then $\mathbb{S}_{\langle\lambda\rangle} V$ is the direct sum of the two irreducible representations of $\mathfrak{s o}_{2 n}$ with highest weight $\lambda_{1} \varepsilon_{1}+\cdots+\lambda_{n-1} \varepsilon_{n-1}+\lambda_{n} \varepsilon_{n}$, and $\lambda_{1} \varepsilon_{1}+$ $\cdots+\lambda_{n-1} \varepsilon_{n-1}-\lambda_{n} \varepsilon_{n}$.

Proof. If $G=\mathrm{Sp}_{2 n}$, this is [24, Thm. 17.11 and Cor. 17.21]. If $G=\mathrm{O}_{2 n}$, this is [24, Thm. 19.19 and Thm. 19.22].

3.4. Lefschetz representations and the generalized Hodge conjecture for abelian varieties of totally real type. The generalized Hodge conjecture was established by Hazama [26] for abelian varieties whose simple factors are of type I or II and whose Hodge group coincides with their Lefschetz group, for $n$-dimensional simple abelian varieties of type I with $n / e$ odd $\left(e=\operatorname{dim}_{\mathbb{Q}} \operatorname{End}^{0}(A)\right)$ by Tankeev [49] (and in particular for odd-dimensional simple abelian varieties of type I), for certain simple abelian varieties of CM-type by Tankeev [49]. Abdulali [1] and Hazama [27] showed that the generalized Hodge conjecture for abelian varieties of CM-type is implied by the Hodge conjecture for the same class of abelian varieties. Here we take a different approach and establish a strong form of the generalized Hodge conjecture for Lefschetz sub-representations of abelian varieties of totally real type.

Theorem 3.7 (GHC for Lefschetz sub-representations of abelian varieties of totally real type). Let $A$ be a complex abelian variety, and let $H \subseteq \mathrm{H}^{k}(A, \mathbb{Q})$ be a Lefschetz sub-representation of Hodge level $\leq k-2 n$. Suppose that $A$ is of totally real type, i.e., that the simple factors of the isogeny class of A have type I, II, or III. Then

$$
H \subseteq \operatorname{Im}\left(\overline{\mathrm{R}}^{n}(A) \otimes \mathrm{H}^{k-2 n}(A, \mathbb{Q}) \stackrel{\cup}{\longrightarrow} \mathrm{H}^{k}(A, \mathbb{Q})\right) .
$$

In fact, we are going to show a stronger statement, namely that the conclusion of Theorem 3.7

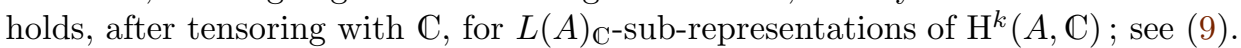

The key point towards the proof of Theorem 3.7 consists in computing the "Hodge level" of the representations $\mathbb{S}_{\langle\lambda\rangle} V$ for $G=\mathrm{Sp}_{2 n}$ or $\mathrm{O}_{2 n}$. Strictly speaking, the spaces $V$ we are going to deal with are not Hodge structures. Rather, as described in Section 3.2, they are complex vector spaces $V_{\sigma}$ endowed with a basis $\left(e_{1}, \ldots, e_{n}, e_{-1}, \ldots, e_{-n}\right)$ and a (skew-)symmetric form $\psi=\sum_{i=1}^{n}\left(e_{i} \otimes e_{-i} \pm e_{-i} \otimes e_{i}\right)$, together with an action of $\mathrm{GL}_{1}$ given by $z \cdot e_{i}=z e_{i}$ and $z \cdot e_{-i}=z^{-1} e_{-i}$ for $1 \leq i \leq n$. Since the action of $\mathrm{GL}_{1}$ on $\psi$ is the identity and since it commutes with the action of permutations in $\mathfrak{S}_{d}$ on $V^{\otimes d}$, the decompositions (5) and (6) commute with the action of $\mathrm{GL}_{1}$. In particular, for a Young tableau $\lambda$ of length $d$, we have a decomposition

$$
\mathbb{S}_{\langle\lambda\rangle} V=\bigoplus_{p+q=d}\left(\mathbb{S}_{\langle\lambda\rangle} V\right)^{p, q},
$$

where

$$
\left(\mathbb{S}_{\langle\lambda\rangle} V\right)^{p, q}:=\left\{w \in \mathbb{S}_{\langle\lambda\rangle} V: z \cdot w=z^{p-q} w \text { for all } z \in \mathrm{GL}_{1}(\mathbb{C})\right\} .
$$

Our Theorem 3.7 generalizes Hazama's [26, Theorem 5.1] by taking into account Lefschetz sub-representations and by including factors of type III. The proof is inspired by loc. cit., but differs from it in that we focus on the representations $\mathbb{S}_{\langle\lambda\rangle} V$ : on the one hand, by Weyl's

\footnotetext{
${ }^{6}$ Representations of associated partitions restricted to $\mathrm{SO}_{2 n}$ are isomorphic. Two partitions (each with the sum of the first two column lengths at most $2 n$ ) are said to be associated if the sum of the lengths of their first columns is $2 n$ and the other columns of their Young diagram have the same lengths.
} 
construction outlined in $\S 3.3$, we completely avoid resorting to understanding the irreducible sub-representations of tensor products as in [26, Lemma 5.1.2]; on the other hand, as explained before Lemma 3.5, these representations $\mathbb{S}_{\langle\lambda\rangle} V$ are not in general the complexifications of subHodge structures, as seems to be assumed in [26, Prop. 4.3]. However, an important feature will be that these irreducible sub-representations are numerically Hodge symmetric.

Lemma 3.8. Let $V$ be an even-dimensional complex vector space with basis $\left(e_{1}, \ldots, e_{n}, e_{-1}, \ldots, e_{-n}\right)$, and assume that $G$ is one of the following groups:

(a) $G=\operatorname{Sp}(V, \psi)$, where $\psi=\sum_{i=1}^{n}\left(e_{i} \otimes e_{-i}-e_{-i} \otimes e_{i}\right)$;

(b) $G=\mathrm{O}(V, \psi)$, where $\psi=\sum_{i=1}^{n}\left(e_{i} \otimes e_{-i}+e_{-i} \otimes e_{i}\right)$ and $n>1$.

Consider the action of the torus $\mathrm{GL}_{1}$ on $V$ given by $z \cdot e_{i}=z e_{i}$ and $z \cdot e_{-i}=z^{-1} e_{-i}$ for $1 \leq i \leq n$. Let $\lambda$ be a Young tableau of length d. Then $\mathbb{S}_{\langle\lambda\rangle} V$ is numerically Hodge symmetric, that is, $\operatorname{dim}_{\mathbb{C}}\left(\mathbb{S}_{\langle\lambda\rangle} V\right)^{p, q}=\operatorname{dim}_{\mathbb{C}}\left(\mathbb{S}_{\langle\lambda\rangle} V\right)^{q, p}$ for all integers $p$ and $q$. Moreover, if $\mathbb{S}_{\langle\lambda\rangle} V \neq 0$, then

$$
\left(\mathbb{S}_{\langle\lambda\rangle} V\right)^{d, 0} \neq 0 .
$$

Proof. Our strategy of proof is taken from Hazama's proof of [26, Prop. 4.3] where the case $G=\mathrm{Sp}_{2 n}$ was treated. Contrary to Hazama, we do not assume that $V$ is the complexification of a Hodge structure (since when extending scalars to $\mathbb{C}$ the irreducible representations of the Lefschetz group that arise are not Hodge structures).

We view $\mathbb{S}_{\langle\lambda\rangle} V$ as a representation of the Lie algebra $\mathfrak{g}$. In both cases $\left(\mathfrak{g}=\mathfrak{s p}_{2 n}\right.$ or $\left.\mathfrak{s o}_{2 n}\right)$, let us recall that, as in Bourbaki [15, Chap. VIII, $\S 13.3 \& \S 13.4$, we let $E_{i, j}$ be the $2 n \times 2 n$ matrix expressed in the basis $\left(e_{1}, \ldots, e_{n}, e_{-1}, \ldots, e_{-n}\right)$ whose entries are all zero except for the $(i, j)$-th entry which is 1 . For $1 \leq i \leq n$, the elements

$$
H_{i}:=E_{i, i}-E_{-i,-i}
$$

define a basis of a Cartan sub-algebra $\mathfrak{h}$ of $\mathfrak{g}$, and we let $\left(\varepsilon_{1}, \ldots, \varepsilon_{n}\right)$ be the dual basis of $\left(H_{1}, \ldots, H_{n}\right)$.

Viewing $\mathbb{S}_{\langle\lambda\rangle} V$ as a subspace of $V^{\otimes d}$, we have the description

$$
\left(\mathbb{S}_{\langle\lambda\rangle} V\right)^{p, q}:=\left\{w \in \mathbb{S}_{\langle\lambda\rangle} V: H_{0}(w)=(p-q) w\right\}, \quad \text { where } H_{0}:=\sum_{i} H_{i} .
$$

In particular, we have $\left(\left(\mathbb{S}_{\langle\lambda\rangle} V\right)^{p, q}\right)^{\vee}=\left(\mathbb{S}_{\langle\lambda\rangle} V^{\vee}\right)^{q, p}$, and since $V \simeq V^{\vee}$ as $\mathfrak{g}$-representations for our Lie algebras $\mathfrak{g}=\mathfrak{s p}_{2 n}$ or $\mathfrak{s o}_{2 n}$, this immediately yields that $\mathbb{S}_{\langle\lambda\rangle} V$ is numerically Hodge symmetric. We also find that $\max \left\{p-q:\left(\mathbb{S}_{\langle\lambda\rangle} V\right)^{p, q} \neq 0\right\}$ is equal to the maximum of the eigenvalues of $H_{0}$ acting on $\mathbb{S}_{\langle\lambda\rangle} V$. We are going to show that if $\mathbb{S}_{\langle\lambda\rangle} V \neq 0$, then $\max \{p-q$ : $\left.\left(\mathbb{S}_{\langle\lambda\rangle} V\right)^{p, q} \neq 0\right\}=d$.

First consider an irreducible representation $W$ of $\mathfrak{g}=\mathfrak{s p}_{2 n}$ or $\mathfrak{s o}_{2 n}$ with highest weight $\omega$. Let $v \in W$ denote one of its dominant vectors. Then for any element $H$ in the Cartan sub-algebra $\mathfrak{h}$ of $\mathfrak{g}$, we have

$$
H(v)=\omega(H) v .
$$

Denote $\alpha_{i}$ the simple roots of $\mathfrak{g}$. Specifically, if $\mathfrak{g}=\mathfrak{s o}_{2 n}$, then $\alpha_{1}=\varepsilon_{1}-\varepsilon_{2}, \ldots, \alpha_{n-1}=$ $\varepsilon_{n-1}-\varepsilon_{n}, \alpha_{n}=2 \varepsilon_{n}$, and if $\mathfrak{g}=\mathfrak{s p}_{2 n}$, then $\alpha_{1}=\varepsilon_{1}-\varepsilon_{2}, \ldots, \alpha_{n-1}=\varepsilon_{n-1}-\varepsilon_{n}, \alpha_{n}=\varepsilon_{n-1}+\varepsilon_{n}$. Since the weights of $W$ are of the form

$$
\omega-\sum_{i=1}^{n} p_{i} \alpha_{i}
$$

for some nonnegative integers $p_{i}$, we find that

$$
\begin{aligned}
\max \left\{p-q:\left(\mathbb{S}_{\langle\lambda\rangle} V\right)^{p, q} \neq 0\right\} & =\max \left\{\left(\omega-\sum_{i} p_{i} \alpha_{i}\right)\left(H_{0}\right): p_{1}, \ldots, p_{n} \geq 0\right\} \\
& =\omega\left(H_{0}\right) .
\end{aligned}
$$

In our case, by Proposition 3.6, $\mathbb{S}_{\langle\lambda\rangle} V$ corresponds either to an irreducible representation of $\mathfrak{g}$ with highest weight $\lambda_{1} \varepsilon_{1}+\cdots+\lambda_{n} \varepsilon_{n}$, or in case $\mathfrak{g}=\mathfrak{s o}_{2 n}$ and $\lambda_{n}>0$ to the sum of two irreducible 
representations with highest weight $\lambda_{1} \varepsilon_{1}+\cdots+\lambda_{n} \varepsilon_{n}$ and $\lambda_{1} \varepsilon_{1}+\cdots+\lambda_{n-1} \varepsilon_{n-1}-\lambda_{n} \varepsilon_{n}$. In any case, we find that $\max \left\{p-q:\left(\mathbb{S}_{\langle\lambda\rangle} V\right)^{p, q} \neq 0\right\}$ is equal to $\omega\left(H_{0}\right)$, where $\omega=\lambda_{1} \varepsilon_{1}+\cdots+\lambda_{n} \varepsilon_{n}$, and hence is equal to $d:=\sum_{i} \lambda_{i}$.

Proof of Theorem 3.7. We first note that it is enough to establish the theorem with complex coefficients. Precisely, we are going to show that for $H \subseteq \mathrm{H}^{k}(A, \mathbb{C})$ a $L(A)_{\mathbb{C}^{-} \text {-sub-representation }}$ of Hodge level $\leq k-2 n$, we have that $H$ is numerically Hodge symmetric and that

$$
H \subseteq \operatorname{Im}\left(\overline{\mathrm{R}}^{n}(A)_{\mathbb{C}} \otimes \mathrm{H}^{k-2 n}(A, \mathbb{C}) \stackrel{\cup}{\longrightarrow} \mathrm{H}^{k}(A, \mathbb{C})\right) .
$$

Here, by Hodge level we mean the following: since $L(A)_{\mathbb{C}}$ contains the circle group (defining

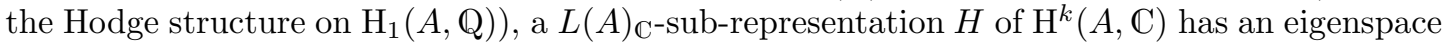
decomposition $\bigoplus_{p+q=k} H^{p, q}$, and the Hodge level is then $\ell(H):=\max \left\{|p-q|: H^{p, q} \neq 0\right\}$. For ease of notation, we write from now on $\mathrm{H}^{*}(-)_{\mathbb{C}}$ for $\mathrm{H}^{*}(-, \mathbb{C})$.

Second, by considering the surjective homomorphism of Lefschetz representations $\mathrm{H}^{1}(A)^{\otimes k} \rightarrow \mathrm{H}^{k}(A)$ given by cup-product, we note that we may assume that $H$ is an irreducible Lefschetz sub-representation of $\mathrm{H}^{1}(A)_{\mathbb{C}}^{\otimes k} \subseteq \mathrm{H}^{k}\left(A^{k}\right)_{\mathbb{C}}$.

Suppose then that $A$ is isogenous to $A_{1}^{m_{1}} \times \cdots \times A_{s}^{m_{s}}$, where the $A_{i}$ are pairwise nonisogenous, simple abelian varieties. The $L(A)_{\mathbb{C}}$-representation $\mathrm{H}^{1}(A)_{\mathbb{C}}$ is isomorphic to the $\left(L\left(A_{1}\right)_{\mathbb{C}} \times \cdots \times L\left(A_{s}\right)_{\mathbb{C}}\right)$-representation $\mathrm{H}^{1}\left(A_{1}\right)_{\mathbb{C}}^{\oplus m_{1}} \oplus \cdots \oplus \mathrm{H}^{1}\left(A_{s}\right)_{\mathbb{C}}^{\oplus m_{s}}$, where each $L\left(A_{i}\right)_{\mathbb{C}}$ acts diagonally on $\mathrm{H}^{1}\left(A_{i}\right)_{\mathbb{C}}^{\oplus m_{i}}$. If $H$ is an irreducible Lefschetz sub-representation of $\mathrm{H}^{1}(A)_{\mathbb{C}}^{\otimes k}$, then up to permutation of the factors we may view $H$ as an irreducible sub-representation of $\mathrm{H}^{1}\left(A_{1}\right)_{\mathbb{C}}^{\otimes k_{1}} \otimes \cdots \otimes \mathrm{H}_{\mathbb{C}}^{1}\left(A_{s}\right)^{\otimes k_{s}}$ for some non-negative integers $k_{i}$ such that $\sum k_{i}=k$. Since $H$ is a $\left(L\left(A_{1}\right)_{\mathbb{C}} \times \cdots \times L\left(A_{s}\right)_{\mathbb{C}}\right)$-sub-representation, $H$ must be of the form

$$
H=H_{1} \otimes \cdots \otimes H_{s}
$$

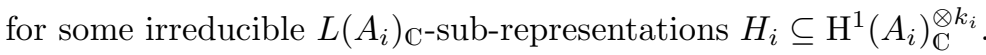

With notations as in $\S 3.2, L\left(A_{i}\right)_{\mathbb{C}}$ is isomorphic to $f:=[F: \mathbb{Q}]$ copies of the group $G$, which is either the symplectic group (types I and II) or the orthogonal group (type III), and $\mathrm{H}^{1}\left(A_{i}\right)_{\mathbb{C}}$ splits as the direct sum of $f$ copies of the standard representation $V$ of $G$ (type I) or as the direct sum of $2 f$ copies of the standard representation $V$ of $G$ (types II and III). Thus $H_{i}$ is an irreducible sub-representation of $V_{1}^{\otimes d_{1}} \otimes \cdots \otimes V_{t}^{\otimes d_{t}}$, where the $j$-th factor of $G^{\times t}=G \times \cdots \times G$ acts on $V_{j}$ as the standard representation and where the other factors act trivially. Hence, $H_{i}$ is of the form

$$
H_{i}=H_{i, 1} \otimes \cdots \otimes H_{i, t}
$$

for some irreducible $G$-sub-representations $H_{i, j} \subseteq V_{j}^{\otimes d_{j}}$.

Now, by Proposition 3.6, each $H_{i, j}$ must be of the form

$$
H_{i, j}=\Psi_{I_{1}} \circ \cdots \circ \Psi_{I_{k_{i, j}}}\left(\mathbb{S}_{\left\langle\lambda_{i, j}\right\rangle} V_{j}\right)
$$

for some Young tableau $\lambda_{i, j}$ and some pairs of integers $I_{1}, \ldots, I_{k_{i, j}}$. From Lemma 3.5, we know that $V_{j}$ decomposes as $V_{j}^{1,0} \oplus V_{j}^{0,1}$ in such a way that both $V_{j}^{1,0}$ and $V_{j}^{0,1}$ are isotropic for the non-degenerate bilinear form on $V_{j}$ (which is skew-symmetric for types I and II, and symmetric for type III). The assumptions of Lemma 3.8 are thus met for $V_{j}$, and we therefore see that $H_{i, j}$ is numerically Hodge symmetric and satisfies $\left(H_{i, j}\right)^{d_{i, j}, 0} \neq 0$, where $d_{i, j}$ is the length of the Young tableau $\lambda_{i, j}$. We deduce that $H$ is numerically Hodge symmetric and satisfies

$$
\ell(H)=\sum_{i, j} d_{i, j}
$$

Now we can conclude, because composing with $\Psi_{I}$ amounts to cupping with a divisor with complex coefficients.

Remark 3.9. Our method for establishing the generalized Hodge conjecture for Lefschetz subrepresentations of abelian varieties of totally real type, which in fact consists in establishing it after extending the scalars to $\mathbb{C}$, is too crude to work for powers of simple abelian varieties of 
type IV. Let us briefly describe a simple example. Beforehand, on a positive note, we simply mention that the method works for powers of a CM elliptic curve. Let then $A$ be a simple abelian surface of type IV; it is known that $A$ must be of CM type, so that its Lefschetz group is $\mathrm{GL}(1) \times \mathrm{GL}(1)$ after extending the scalars to $\mathbb{C}$ and we may write $\mathrm{H}^{1}(A, \mathbb{C})=(V \oplus$ $\left.V^{\vee}\right) \oplus\left(W \oplus W^{\vee}\right)$, where $V=V^{1,0}$ and $W=W^{1,0}$ and $\operatorname{GL}(1) \times \operatorname{GL}(1)$ acts on $V$ via the first projection and on $W$ via the second projection. Consider then for instance the 1-dimensional

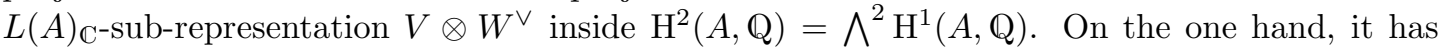
Hodge type $(1,1)$ but is not acted upon trivially by $L(A)_{\mathbb{C}}$ and thus is not spanned by a Hodge class. That type of phenomenon does not occur for abelian varieties of totally real type because their Lefschetz representations are numerically Hodge symmetric (Lemma 3.5). On the other hand, we deduce that the Galois orbit of $V \otimes W^{\vee}$ inside $\mathrm{H}^{2}(A, \mathbb{Q})$ has Hodge length 2 ; this suggests that it is not straightforward to read the Hodge length of the Galois closure of a

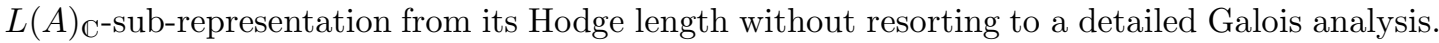

3.5. Lefschetz representations and the generalized Hodge conjecture II. In this section, we would like to improve slightly on Theorem 3.7 by allowing our abelian varieties to be isogenous to the product of an abelian variety of totally real type with some power of an abelian surface of CM type, or with the product of powers of three elliptic curves. Our main result is Proposition 3.12. In particular, we recall a strong version of the generalized Hodge conjecture for self-powers of abelian surfaces; see Corollary 3.13.

Let us start with the case where our abelian varieties have no factor of totally real type. The following theorem is due to Abdulali [1, Examples 2 \& 3]:

Theorem 3.10 (Abdulali [1], strong GHC for powers of CM abelian surfaces and certain products of CM elliptic curves). Let $A$ be an abelian variety that is isogenous to either

(i) $E_{1}^{k_{1}} \times E_{2}^{k_{2}} \times E_{3}^{k_{3}}$ for some $C M$ elliptic curves $E_{i}$, or

(ii) the power of a $C M$ abelian surface $S$.

Let $H \subseteq \mathrm{H}^{k}(A, \mathbb{Q})$ be a Hodge sub-structure of Hodge level $\leq k-2 n$. Then

$$
H \subseteq \sum_{B} \operatorname{Im}\left(\mathrm{R}^{\operatorname{dim} B+n}(A \times B) \otimes \mathrm{H}^{k-2 n}(B, \mathbb{Q}) \longrightarrow \mathrm{H}^{k}(A, \mathbb{Q})\right),
$$

where $\Gamma \otimes \gamma \mapsto \Gamma^{*}(\gamma)$ and where the sum runs over all abelian varieties $B$.

Proof. For a proof, we refer to Abdulali [1]. Let us mention that in case $(i)$ the sum can be taken over abelian varieties of the form $E_{1}^{m_{1}} \times E_{2}^{m_{2}} \times E_{3}^{m_{3}}$, and in case $(i i)$ over powers of $S$, unless $S$ is an abelian surface with $\mathrm{CM}$ by a field $E$ not Galois over $\mathbb{Q}$, in which case, denoting $S^{\prime}$ the other abelian surface with CM by $E$, the sum runs through abelian varieties of the form $S^{i} \times\left(S^{\prime}\right)^{j}$. That the correspondences in the sum can be chosen to be in $\mathrm{R}^{*}$ is due to the fact that for abelian varieties of the form $E_{1}^{m_{1}} \times E_{2}^{m_{2}} \times E_{3}^{m_{3}}$, or $S^{i} \times\left(S^{\prime}\right)^{j}$ as above, the Hodge group coincides with the Lefschetz group, so that all Hodge classes on $E_{1}^{m_{1}} \times E_{2}^{m_{2}} \times E_{3}^{m_{3}}$ or $S^{i} \times\left(S^{\prime}\right)^{j}$ belong in fact to $\overline{\mathrm{R}}^{*}$.

Proposition 3.11. We have the following three statements:

(a) Let $A$ be an abelian variety and let $m$ be a positive integer. Then any symmetrically distinguished cycle on $A^{m}$ that is generically defined for $m$-fold powers of polarized abelian varieties belongs to $\mathrm{R}^{*}\left(A^{m}\right)$.

(b) Let $A, B$ and $C$ be abelian varieties, and let $\gamma \in \mathrm{R}^{*}(A \times B)$ and $\gamma^{\prime} \in \mathrm{R}^{*}(B \times C)$ be two correspondences. Then $\gamma^{\prime} \circ \gamma$ belongs to $\mathrm{R}^{*}(A \times C)$.

(c) Let $f: A \rightarrow B$ be a homomorphism of abelian varieties. Then the graph $\Gamma_{f}$ of $f$ belongs to $\mathrm{R}^{*}(A \times B)$.

Proof. By O'Sullivan's Theorem 1.2, a symmetrically distinguished cycle in $\mathrm{DCH}^{*}(A)$ whose cohomology class belongs to $\overline{\mathrm{R}}^{*}(A)$ belongs to $\mathrm{R}^{*}(A)$ for any abelian variety $A$. Thus (a) follows from the fact that the cohomology class of a generically defined cycle on the $m$-fold 
power of a polarized abelian variety $A$ belongs to $\bar{R}^{*}\left(A^{m}\right)$; see Theorem 2.12. (More precisely, Hodge classes on $A^{m}$, with $A$ a very general polarized abelian variety, consist of polynomials in $p_{i}^{*} L$ and $p_{i, j}^{*} c_{1}\left(\mathcal{P}_{\mathcal{A}}\right)$, where $L$ is the polarization of $A$.)

For (b), observe that the composition of two correspondences in $\overline{\mathrm{R}}^{*}$ yields a correspondence in $\overline{\mathrm{R}}^{*}$; indeed a correspondence belongs to $\overline{\mathrm{R}}^{*}$ if and only if it commutes with the action of the Lefschetz group. Case (b) then follows from this fact together with the fact that by O'Sullivan's Theorem $\gamma^{\prime} \circ \gamma$ is symmetrically distinguished (since $\gamma$ and $\gamma^{\prime}$ are).

For (c), since $\Gamma_{f}=\left(f, \operatorname{id}_{B}\right)^{*} \Delta_{B}$, it suffices to show that $\Delta_{B} \in \mathrm{R}^{*}(B \times B)$. This can be found in $[47, \S 5]$.

As a consequence of Theorems 3.7 and 3.10, we have the following analogue of Proposition 2.13, which in particular establishes Conjecture 1.6 for Lefschetz sub-representations of certain abelian varieties:

Proposition 3.12. Let $A$ be a complex abelian variety of dimension g, and let $H \subseteq \mathrm{H}^{k}(A, \mathbb{Q})$ be a Lefschetz sub-representation of Hodge level $\leq k-2 n$. Assume that $A$ is isogenous to $A_{0} \times A_{1}$ with

- $A_{0}$ isomorphic to $E_{1}^{k_{1}} \times E_{2}^{k_{2}} \times E_{3}^{k_{3}}$ for some $C M$ elliptic curves $E_{i}$, or to the power of a $C M$ abelian surface;

- $A_{1}$ isomorphic to an abelian variety of totally real type (cf. Definition 1).

Then there exists an idempotent correspondence $p_{H} \in \mathrm{R}^{g}(A \times A)$ inducing the projection $\mathrm{H}^{*}(A, \mathbb{Q}) \rightarrow H \rightarrow \mathrm{H}^{*}(A, \mathbb{Q})$, which is a linear combination of correspondences of the form

$$
\mathfrak{h}(A) \stackrel{\rho}{\longrightarrow} \mathfrak{h}(B)(n) \stackrel{\zeta}{\longrightarrow} \mathfrak{h}(A),
$$

for some abelian varieties $B$ and some correspondences $\rho$ and $\zeta$ that belong to $\mathrm{R}^{*}(A \times B)$ and $\mathrm{R}^{*}(B \times A)$, respectively.

Proof. First we show a strong version of the generalized Hodge conjecture for Lefschetz subrepresentations of $A$; namely, we show that

$$
H \subseteq \sum_{B} \operatorname{Im}\left(\mathrm{R}^{\operatorname{dim} B+n}(A \times B) \otimes \mathrm{H}^{k-2 n}(B, \mathbb{Q}) \longrightarrow \mathrm{H}^{k}(A, \mathbb{Q})\right),
$$

where $\Gamma \otimes \gamma \mapsto \Gamma^{*}(\gamma)$ and where the sum runs over all abelian varieties $B$. As outlined after the proof of [2, Prop. 4] in the context of Hodge sub-structures, there is a slight subtlety: one needs to use the stronger statement of Theorem 3.7 described in its proof, namely, that for $H_{1} \subseteq \mathrm{H}^{k_{1}}\left(A_{1}, \mathbb{C}\right)$ a $L\left(A_{1}\right)_{\mathbb{C}^{-} \text {-sub-representation of level }} \leq k_{1}-2 n_{1}$, we have that $H_{1}$ is numerically Hodge symmetric and that

$$
H_{1} \subseteq \operatorname{Im}\left(\overline{\mathrm{R}}^{n_{1}}\left(A_{1}\right)_{\mathbb{C}} \otimes \mathrm{H}^{k_{1}-2 n_{1}}\left(A_{1}, \mathbb{C}\right) \stackrel{\cup}{\longrightarrow} \mathrm{H}^{k_{1}}\left(A_{1}, \mathbb{C}\right)\right) .
$$

Let $H$ be an irreducible Lefschetz sub-representation of $\mathrm{H}^{k}\left(A_{0} \times A_{1}, \mathbb{Q}\right)$. Then $H$ is a subrepresentation of $L\left(A_{0} \times A_{1}\right) \cong L\left(A_{0}\right) \times L\left(A_{1}\right)$ acting on some Künneth component $\mathrm{H}^{k_{0}}\left(A_{0}, \mathbb{Q}\right) \otimes$ $\mathrm{H}^{k_{1}}\left(A_{1}, \mathbb{Q}\right)$ for some $k_{0}+k_{1}=k$. Let then $V$ be an irreducible sub-representation of $L\left(A_{0} \times A_{1}\right)_{\mathbb{C}}$

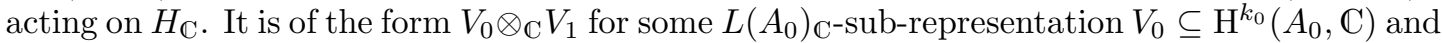

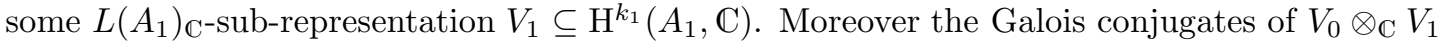
span $H_{\mathbb{C}}$; indeed, the span is defined over $\mathbb{Q}$ and defines a non-trivial sub-representation of the irreducible $L\left(A_{0} \times A_{1}\right)$-representation $H$. The subspace spanned by the Galois conjugates of $V_{0}$ inside $\mathrm{H}^{k_{0}}\left(A_{0}, \mathbb{C}\right)$ is defined over $\mathbb{Q}$; we denote it $W_{0}$. Then $W_{0}$ is a $L\left(A_{0}\right)$-sub-representation of $\mathrm{H}^{k_{0}}\left(A_{0}, \mathbb{Q}\right)$.

\footnotetext{
${ }^{7}$ See the proof of Theorem 3.7 for the notion of level of a $L\left(A_{1}\right)_{\mathbb{C}^{-}}$sub-representation.
} 
We note from Theorem 3.7 and its proof that $V_{1}$ and its Galois conjugates $V_{1}^{\sigma}$ are Hodge symmetric of same level. We find

$$
\begin{aligned}
\ell(H) & =\max _{\sigma} \ell\left(V_{0}^{\sigma} \otimes V_{1}^{\sigma}\right) \\
& =\max _{\sigma}\left(\ell\left(V_{0}^{\sigma}\right)+\ell\left(V_{1}^{\sigma}\right)\right) \\
& =\max _{\sigma} \ell\left(V_{0}^{\sigma}\right)+\ell\left(V_{1}\right) \\
& =\ell\left(W_{0}\right)+\ell\left(V_{1}\right) .
\end{aligned}
$$

Here the maximum is taken over all elements $\sigma \in \operatorname{Aut}_{\mathbb{Q}}(\mathbb{C})$, and the second equality holds because $V_{1}^{\sigma}$ is Hodge symmetric. Let us then write

$$
\ell\left(W_{0}\right)=k_{0}-2 n_{0} \quad \text { and } \quad \ell\left(V_{1}\right)=k_{1}-2 n_{1}, \quad \text { for } n_{1}+n_{2}=n .
$$

By the above (11), there are an integer $s$ and correspondences $\Gamma_{r, 1} \in \mathrm{R}^{*}\left(A_{1} \times A_{1}\right)_{\mathbb{C}}, 1 \leq r \leq s$, such that

$$
V_{1} \subseteq \sum_{r} \Gamma_{r, 1, *} \mathrm{H}^{k_{1}-2 n_{1}}\left(A_{1}, \mathbb{C}\right)
$$

Since each $\Gamma_{r, 1}$ is a $\mathbb{C}$-linear combination of elements in $\mathrm{R}^{*}\left(A_{1} \times A_{1}\right)$, up to increasing $s$, we may assume that each $\Gamma_{r, 1}$ is in fact in the image of $\mathrm{R}^{*}\left(A_{1} \times A_{1}\right) \hookrightarrow \mathrm{R}^{*}\left(A_{1} \times A_{1}\right)_{\mathbb{C}}$, so that for every $\sigma \in \operatorname{Aut}(\mathbb{C})$, we have

$$
V_{1}^{\sigma} \subseteq \sum_{r} \Gamma_{r, 1, *} \mathrm{H}^{k_{1}-2 n_{1}}\left(A_{1}, \mathbb{C}\right) .
$$

On the other hand, there are finitely many non-zero correspondences $\Gamma_{B, 0} \in \mathrm{R}^{*}\left(B \times A_{0}\right)$ indexed by abelian varieties $B$, such that

$$
W_{0} \subseteq \sum_{B} \Gamma_{B, 0, *} \mathrm{H}^{k_{0}-2 n_{0}}(B, \mathbb{Q}) .
$$

Since $V_{0} \subseteq W_{0, \mathbb{C}}$, we have

$$
\begin{aligned}
H_{\mathbb{C}} & =\sum_{\sigma \in \operatorname{Aut}(\mathbb{C})}\left(V_{0} \otimes_{\mathbb{C}} V_{1}\right)^{\sigma} \subseteq \sum_{\sigma, \tau \in \operatorname{Aut}(\mathbb{C})}\left(V_{0}^{\sigma} \otimes_{\mathbb{C}} V_{1}^{\tau}\right)=W_{0, \mathbb{C}} \otimes \sum_{\tau \in \operatorname{Aut}(\mathbb{C})} V_{1}^{\tau} \\
& \subseteq\left(\sum_{B} \Gamma_{B, 0, \mathbb{C}, *} \mathrm{H}^{k_{0}-2 n_{0}}(B, \mathbb{C})\right) \otimes\left(\sum_{r} \Gamma_{r, 1, \mathbb{C}, *} \mathrm{H}^{k_{1}-2 n_{1}}\left(A_{1}, \mathbb{C}\right)\right) \\
& \subseteq \sum_{B, r}\left(\Gamma_{B, 0} \otimes \Gamma_{r, 1}\right)_{\mathbb{C}, *} \mathrm{H}^{k_{0}+k_{1}-2\left(n_{0}+n_{1}\right)}\left(B \times A_{1}, \mathbb{C}\right) .
\end{aligned}
$$

This establishes (10).

Now, since $H$ is a Lefschetz sub-representation of $\mathrm{H}^{k}(A, \mathbb{Q})$, there exists by Lemma 3.4 an idempotent $\pi_{H} \in \mathrm{R}^{g}(A \times A)$ such that $\left(\pi_{H}\right)_{*} \mathrm{H}^{k}(A, \mathbb{Q})=H$. Composing $\pi_{H}$ with the correspondence $\sum \Gamma_{B, 0} \otimes \Gamma_{r, 1}$, we see that

$$
H=\left(\pi_{H} \circ \sum_{B, r} \Gamma_{B, 0} \otimes \Gamma_{r, 1}\right)_{*} \mathrm{H}^{k-2 n}\left(B \times A_{1}, \mathbb{Q}\right) .
$$

In order to conclude the proof of Proposition 3.12, we observe that we may proceed as in the proof of Proposition 2.13; indeed, all the correspondences appearing there are compositions of correspondences in $\mathrm{R}^{*}$, and therefore thanks to Proposition 3.11 belong to $\mathrm{R}^{*}$.

As a corollary, let us mention the following result, $c f .[3,8.1(2)]$.

Corollary 3.13 (strong GHC for self-powers of elliptic curves, or abelian surfaces). Let $A$ be an abelian variety of dimension $\leq 2$, and let $m$ be a positive integer. Let $H \subseteq \mathrm{H}^{k}\left(A^{m}, \mathbb{Q}\right)$ be a Hodge sub-structure of Hodge level $\leq k-2 n$. Then

$$
H \subseteq \sum_{B} \operatorname{Im}\left(\mathrm{R}^{\operatorname{dim} B+n}\left(A^{m} \times B\right) \otimes \mathrm{H}^{k-2 n}(B, \mathbb{Q}) \longrightarrow \mathrm{H}^{k}\left(A^{m}, \mathbb{Q}\right)\right),
$$

where $\Gamma \otimes \gamma \mapsto \Gamma^{*}(\gamma)$ and where the sum runs over all abelian varieties $B$. 
Proof. The case where $A$ has CM was covered in Abdulali's Theorem 3.10, while the case where $A$ is without CM is covered by Theorem 3.7 (recall that in these cases, $\operatorname{Hdg}(A)=L(A)$ ). Thus it only remains to treat the case where $A=E \times E^{\prime}$, where $E$ is an elliptic curve without CM and $E^{\prime}$ is an elliptic curve with CM. In that case, we still have $\operatorname{Hdg}(A)=L(A)$ (see e.g. [36]) and one concludes with Proposition 3.12.

3.6. Lefschetz representations and the generalized Bloch conjecture. We are now in a position to prove the theorem announced in $\S 0.2$ of the introduction.

Theorem 3.14. Let $A$ and $A^{\prime}$ be two abelian varieties, and let $\gamma$ be a cycle in $\mathrm{R}^{*}\left(A \times A^{\prime}\right)$. Assume that $A$ is isogenous to $A_{0} \times A_{1}$ with

- $A_{0}$ isomorphic to $E_{1}^{k_{1}} \times E_{2}^{k_{2}} \times E_{3}^{k_{3}}$ for some $C M$ elliptic curves $E_{i}$, or to the power of a $C M$ abelian surface;

- $A_{1}$ isomorphic to an abelian variety of totally real type (cf. Definition 1).

If $\gamma^{*} \mathrm{H}^{i, j}\left(A^{\prime}\right)=0$ for all $j<n$, then $\gamma_{*} \mathrm{CH}_{r}(A)=0$ for all $r<n$.

Proof. Since $\gamma$ is a cycle in $\mathrm{R}^{*}\left(A \times A^{\prime}\right)$, we have that $\gamma^{*} \mathrm{H}^{*}\left(A^{\prime}, \mathbb{Q}\right)$ is a Lefschetz sub-representation $H$ of $\mathrm{H}^{*}(A, \mathbb{Q})$. By the assumption $\gamma^{*} \mathrm{H}^{i, j}\left(A^{\prime}\right)=0$ for all $j<n$ and by Proposition 3.12, we see that, modulo homological equivalence, $\gamma=\gamma \circ p_{H}$ is a linear combination of cycles in $\mathrm{R}^{*}\left(A \times A^{\prime}\right)$ that factor as

$$
\mathfrak{h}(A) \stackrel{\rho}{\longrightarrow} \mathfrak{h}(B)(n) \stackrel{\zeta}{\longrightarrow} \mathfrak{h}\left(A^{\prime}\right),
$$

for some abelian varieties $B$ and some correspondences $\rho$ and $\zeta$ that belong to $\mathrm{R}^{*}(A \times B)$ and $\mathrm{R}^{*}\left(B \times A^{\prime}\right)$, respectively. Since all the correspondences involved belong to $\mathrm{R}^{*}(-)$, O'Sullivan's Theorem 1.2 tells us that the latter in fact holds modulo rational equivalence. It follows that $\gamma$ factors through a morphism $\mathfrak{h}(A) \rightarrow \bigoplus_{B} \mathfrak{h}(B)(n)$, where the direct sum runs through the abelian varieties that appeared above. In particular, the map $\gamma_{*}: \mathrm{CH}_{r}(A) \rightarrow \mathrm{CH}_{*}\left(A^{\prime}\right)$ factors through a map $\mathrm{CH}_{r}(A) \rightarrow \bigoplus_{B} \mathrm{CH}_{r-n}(B)$, and hence $\gamma_{*}: \mathrm{CH}_{r}(A) \rightarrow \mathrm{CH}_{*}\left(A^{\prime}\right)$ is zero for $r<n$.

Remark 3.15. In the case where $A$ is isogenous to the power of an abelian variety of dimension $\leq 2$, we will use Corollary 3.13 to prove in Theorem 4.7 that if $\gamma$ is a cycle in $\mathrm{CH}^{*}(A \times A)$ such that $\gamma^{*} \mathrm{H}^{i, j}(A)=0$ for all $j<n$, then $\gamma_{*}$ acts nilpotently on $\mathrm{CH}_{r}(A)$ for all $r<n$.

\section{Applications}

The simplest form of Bloch's conjecture predicts that if a smooth projective complex variety $X$ satisfies $h^{i, 0}(X)=0$ for all positive integers $i$, then $\mathrm{CH}_{0}(X)=\mathbb{Q}$. If now $S$ is a smooth projective complex surface that satisfies $h^{1,0}(S)=0$ and $h^{2,0}(S)=1$, then since $\bigwedge^{2} h^{2,0}(S)=0$ one would expect that $a \times b=b \times a$ in $\mathrm{CH}_{0}(S \times S)$ for all zero-cycles $a, b \in \mathrm{CH}_{0}(S)_{\text {num }}$, where $\mathrm{CH}_{0}(S)_{\text {num }}$ denotes the zero-cycles of degree zero. This expectation was studied by Voisin in [53] who conjectured it for K3 surfaces, and established it for Kummer surfaces and for a certain 10-dimensional family of K3 surfaces; see also [31, 32]. Another prediction of Bloch's conjecture is the following. Let $f: X \rightarrow X$ be an automorphism of a smooth projective variety such that $f^{*}$ acts as the identity on $\mathrm{H}^{0}\left(\Omega_{X}^{i}\right)$ for all $i$; then $f$ should act unipotently on $\mathrm{CH}_{0}(X)$. This was checked for finite-order automorphisms of K3 surfaces by Voisin [54] and Huybrechts [28].

In this section, we answer questions of that type for curves, abelian varieties, Kummer surfaces and generalized Kummer varieties. In $\S \S 4.1,4.2$ and 4.3 , we use our results on generically defined cycles, while in $\S \S 4.4$ and 4.5 , we use the strong form of the generalized Hodge conjecture for powers of abelian surfaces.

4.1. Symmetric and skew-symmetric cycles on powers of curves or of abelian varieties. Recall from Shermenev [45] and Deninger-Murre [17] that the Chow motive of an abelian variety $A$ of dimension $g$ admits a weight decomposition

$$
\mathfrak{h}(A)=\bigoplus_{i=0}^{2 g} \mathfrak{h}^{i}(A)
$$


with the property that

$$
\mathrm{CH}^{j}\left(\mathfrak{h}^{i}(A)\right)=\left\{a \in \mathrm{CH}^{i}(A):[n]^{*} a=n^{i} a \text { for all } n \in \mathbb{Z}\right\},
$$

where $[n]: A \rightarrow A$ is the multiplication-by- $n$ homomorphism, and the property that the diagonal embedding $A \hookrightarrow A^{i}$ induces a canonical isomorphism

$$
\mathfrak{h}^{i}(A) \cong \mathrm{S}^{i} \mathfrak{h}^{1}(A)
$$

where the right-hand term denotes the $i$-th symmetric power of the motive $\mathfrak{h}^{1}(A)$, seen as a direct summand of the motive of $A^{i}$.

The following result generalizes to integers $i \neq g$ a result of Voisin [56, Example 4.40]. Note that in the proof of loc. cit., one has to check that $\sigma$ sends $\mathfrak{h}^{g}(A) \otimes \mathfrak{h}^{g}(A)$ into $\mathfrak{h}^{g}(A) \otimes \mathfrak{h}^{g}(A)$ (a priori $\sigma$ sends $\mathfrak{h}^{g}(A) \otimes \mathfrak{h}^{g}(A)$ into $\mathfrak{h}^{2 g}(A \times A)=\bigoplus_{i} \mathfrak{h}^{i}(A) \otimes \mathfrak{h}^{2 g-i}(A)$ ).

Theorem 4.1. Let $A$ be an abelian variety of dimension $g$. Let $i$ be a nonnegative integer.

- For $i$ odd, we have $\mathrm{CH}_{0}\left(\mathrm{~S}^{N} \mathfrak{h}^{2 g-i}(A)\right)=0$ for all $N>\left(\begin{array}{l}g \\ i\end{array}\right)$.

- For $i$ even, we have $\mathrm{CH}_{0}\left(\bigwedge^{N} \mathfrak{h}^{2 g-i}(A)\right)=0$ for all $N>\left(\begin{array}{l}g \\ i\end{array}\right)$.

In particular, if $N>\left(\begin{array}{c}g \\ i\end{array}\right)$ and if $a_{j}, 1 \leq j \leq N$, are zero-cycles on $A$ such that $[n]_{*} a_{j}=n^{i} a_{j}$ for all integers $n$, then the following holds.

- For $i$ odd, we have $\sum_{\sigma \in \mathfrak{S}_{N}} a_{\sigma(1)} \times \cdots \times a_{\sigma(N)}=0$ in $\mathrm{CH}_{0}\left(A^{N}\right)$.

- For $i$ even, we have $\sum_{\sigma \in \mathfrak{S}_{N}} \operatorname{sgn}(\sigma) a_{\sigma(1)} \times \cdots \times a_{\sigma(N)}=0$ in $\mathrm{CH}_{0}\left(A^{N}\right)$.

Proof. The reason for considering symmetric or anti-symmetric powers when $i$ is odd or even, respectively, is because the cohomology ring of a smooth variety is graded-commutative. As for the second part of the theorem, this follows simply from the description (12) of $\mathrm{CH}_{0}\left(\mathfrak{h}^{2 g-i}(A)\right)$.

Given a permutation $\sigma \in \mathfrak{S}_{N}$, let us denote $\Gamma_{\sigma} \in \mathrm{CH}^{N g}\left(A^{N} \times A^{N}\right)$ the graph of the morphism $\left(x_{1}, \ldots, x_{n}\right) \rightarrow\left(x_{\sigma^{-1}(1)}, \ldots, x_{\sigma^{-1}(n)}\right)$. The symmetric projector and the alternate projector are respectively

$$
p_{S^{N}}:=\frac{1}{n !} \sum_{\sigma \in \mathfrak{S}_{N}} \Gamma_{\sigma} \quad \text { and } \quad p_{\wedge^{N}}:=\frac{1}{n !} \sum_{\sigma \in \mathfrak{S}_{N}} \operatorname{sgn}(\sigma) \Gamma_{\sigma} ;
$$

they are generically defined idempotent correspondences for $N$-fold products of polarized abelian varieties of dimension $g$. For $i$ odd, the generically defined correspondence $p_{S^{N}} \circ\left(\pi_{A}^{2 g-i} \otimes\right.$ $\left.\cdots \otimes \pi_{A}^{2 g-i}\right)$ acts trivially on $\mathrm{H}^{N(2 g-i), 0}\left(A^{N}\right)$ for $N>\left(\begin{array}{l}g \\ i\end{array}\right)$. For $i$ even, the generically defined correspondence $p_{\wedge^{N}} \circ\left(\pi_{A}^{2 g-i} \otimes \cdots \otimes \pi_{A}^{2 g-i}\right)$ acts trivially on $\mathrm{H}^{N(2 g-i), 0}\left(A^{N}\right)$ for $N>\left(\begin{array}{l}g \\ i\end{array}\right)$. In both case, we conclude by invoking Theorem 1 .

Remark 4.2. Of course, one can state and prove many variants of Theorem 4.1. For example, given integers $n \leq i$ with say $i$ odd, since the Hodge numbers

$$
h^{N(2 g-i), 0}, h^{N(2 g-i)-1,1}, \ldots, h^{N(2 g-i)-n, n}
$$

of $\mathrm{S}^{N} \mathfrak{h}^{2 g-i}(A)$ vanish for $N>\sum_{j=0}^{n}\left(\begin{array}{c}g \\ j\end{array}\right)\left(\begin{array}{c}g \\ i-j\end{array}\right)$, we can prove that $\mathrm{CH}_{r}\left(\mathrm{~S}^{N} \mathfrak{h}^{2 g-i}(A)\right)=0$ for all $r \leq n$. One could also consider the motives $\bigwedge^{M} S^{N} \mathfrak{h}^{2 g-i}(A)$, various images under Schur functors, etc. Via the Abel-Jacobi map, one also recovers the fact that for a smooth projective curve $C$ of genus $g$ we have

$$
\sum_{\sigma \in \mathfrak{S}_{N}} a_{\sigma(1)} \times \cdots \times a_{\sigma(N)}=0 \text { in } \mathrm{CH}_{0}\left(C^{N}\right),
$$

for any integer $N>g$ and any degree- 0 zero-cycles $a_{1}, \ldots, a_{N} \in \mathrm{CH}_{0}(C)$. This is originally due independently to Voisin [53, p.267] and Voevodsky [51]; since algebraically trivial cycles are parametrized by curves, this establishes that, for any smooth projective variety $X$, any algebraically trivial cycle $a \in \mathrm{CH}^{r}(X)$ is smash-nilpotent, that is, $a \times \cdots \times a=0 \in \mathrm{CH}^{r N}\left(X^{N}\right)$ for some $N>0$. 
4.2. Zero-cycles on generalized Kummer varieties. Let $A$ be an abelian surface. The $n$-th generalized Kummer variety $K_{n}(A)$ associated to $A$ is a fiber of the isotrivial fibration $\operatorname{Hilb}^{n+1}(A) \rightarrow A$ that is the composite of the Hilbert-Chow morphism $\operatorname{Hilb}^{n+1}(A) \rightarrow$ $A^{n+1} / \mathfrak{S}_{n+1}$ with the sum morphism $\Sigma: A^{n+1} / \mathfrak{S}_{n+1} \rightarrow A$. The variety $K_{n}(A)$ is known to be a hyperKähler variety [9], in particular $h^{2 i, 0}\left(K_{n}(A)\right)=1$ for $0 \leq i \leq n$, and $h^{2 i+1,0}\left(K_{n}(A)\right)=0$ for all $i$. A generalized Kummer variety of dimension 2 is nothing but a Kummer surface.

In [22], we established that the Chow ring $\mathrm{CH}^{*}\left(K_{n}(A)\right)$ of generalized Kummer varieties admits a grading that splits the conjectural Bloch-Beilinson filtration. We write

$$
\mathrm{CH}^{*}\left(K_{n}(A)\right)=\bigoplus_{j} \mathrm{CH}^{*}\left(K_{n}(A)\right)_{(j)} .
$$

In the case of zero-cycles, this grading has the following simple description (see [33]). The restriction of the Hilbert-Chow morphism provides a birational morphism from $K_{n}(A)$ to the variety $A_{0}^{n+1} / \mathfrak{S}_{n+1}$, where $A_{0}^{n+1}$ is the fiber over 0 of the sum morphism $\Sigma: A^{n+1} \rightarrow A$ and the action of the symmetric group $\mathfrak{S}_{n+1}$ is the one induced from the action on $A^{n+1}$ permuting the factors. Then $\mathrm{CH}_{0}\left(K_{n}(A)\right)_{(j)}$ identifies with $\left(\mathrm{CH}_{0}\left(A_{0}^{n+1}\right)_{(j)}\right)^{\mathfrak{S}_{n+1}}$ via the restriction of the Hilbert-Chow morphism, where $\mathrm{CH}_{0}\left(A_{0}^{n+1}\right)_{(j)}$ is defined in (2). Let us identify $A_{0}^{n+1}$ with $A^{n}$, and let us write

$$
p:=\frac{1}{(n+1) !} \sum_{\sigma \in \mathfrak{S}_{n+1}} \Gamma_{\sigma}
$$

for the projector on the $\mathfrak{S}_{n+1}$-invariant part of the motive of $A^{n}$; it is a generically defined correspondence for $n$-fold products of polarized abelian surfaces. Then we have

$$
\mathrm{CH}_{0}\left(K_{n}(A)\right)_{(j)}=p^{*} \mathrm{CH}_{0}\left(A^{n}\right)_{(j)}=\left(\pi_{A^{n}}^{j} \circ p\right)^{*} \mathrm{CH}_{0}\left(A^{n}\right),
$$

where $\pi_{A^{n}}^{j}$ is a Chow-Künneth projector as in Lemma 2.6, in particular generically defined.

The following theorem is due to Hsueh-Yung Lin. We provide a short proof based on our Theorem 1.

Theorem $4.3(\operatorname{Lin}[33,34]) \cdot \mathrm{CH}_{0}\left(K_{n}(A)\right)_{(2 j+1)}=0$ for all integers $j$.

Proof. We know that $p^{*} \mathrm{H}^{2 j+1,0}\left(A^{n}\right)=\mathrm{H}^{2 j+1,0}\left(K_{n}(A)\right)=0$ for all integers $j$, so that $\left(\pi_{A^{n}}^{2 j+1}\right.$ 。 $p)^{*} \mathrm{H}^{*}\left(A^{n}, \mathbb{Q}\right) \subseteq \mathrm{N}_{H}^{1} \mathrm{H}^{*}\left(A^{n}, \mathbb{Q}\right)$. The theorem is then a straightforward application of Theorem 1.

The following theorem generalizes a result of Voisin [53, Proposition 3.2] for Kummer surfaces to the higher dimensional case of generalized Kummer varieties.

Theorem 4.4. Let $a$ and $b$ be two cycles in $\mathrm{CH}_{0}\left(K_{n}(A)\right)_{(2 j)}$. Then

$$
a \times b=b \times a \quad \text { in } \mathrm{CH}_{0}\left(K_{n}(A) \times K_{n}(A)\right) .
$$

Proof. Let $p_{\wedge^{2}}$ be the generically defined idempotent $\Delta_{A^{n}}-\Gamma_{\tau} \in \mathrm{CH}^{2 n}\left(A^{n} \times A^{n}\right)$ where $\tau: A^{n} \times$ $A^{n} \rightarrow A^{n} \times A^{n}$ is the morphism permuting the factors. Since $\mathrm{H}^{2 j, 0}\left(A^{n}\right)^{\mathfrak{S}_{n+1}}=\mathrm{H}^{2 j, 0}\left(K_{n}(A)\right)=$ 1, we have $\left(p_{\wedge^{2}} \circ \pi_{A^{n}}^{2 j} \circ p\right)^{*} \mathrm{H}^{*}\left(A^{n}, \mathbb{Q}\right) \subseteq \mathrm{N}_{H}^{1} \mathrm{H}^{*}\left(A^{n}, \mathbb{Q}\right)$. We may now conclude by invoking Theorem 1.

4.3. On a conjecture of Voisin. Let $N \geq 2$ be an integer, and let $S$ be a K3 surface. Denote $p r: S^{N} \rightarrow S^{N-1}$ the projection to the first $N-1$ factors; it induces for all $l \geq 0$ a morphism

$$
p r_{*}:\left(p_{\wedge^{N}}\right)_{*} \mathrm{CH}_{l}\left(S^{N}\right) \longrightarrow\left(p_{\wedge^{N-1}}\right)_{*} \mathrm{CH}_{l}\left(S^{N-1}\right),
$$

where $p_{\wedge^{N}}$ is the anti-symmetrization projector defined in (13). As a consequence of the BlochBeilinson philosophy, Voisin [53, Conjecture 3.9] stated:

Conjecture 4.5 (Voisin). The anti-symmetrization projector $p_{\wedge^{N+1}}$ acts as zero on $\operatorname{ker}\left(p r_{*}\right) \otimes$ $\mathrm{CH}_{0}(S)_{\text {num }}$ for all $l<N$. 
Voisin established this conjecture for $N=2$ in the case where $S$ is a Kummer surface by a lengthy calculation; see [53, Theorem 3.10]. A variant of our Theorem 1 makes it possible to prove (a stronger form of) Voisin's conjecture for Kummer surfaces for all values of $N$.

Theorem 4.6. Conjecture 4.5 is true for Kummer surfaces for all integers $N \geq 2$.

Proof. Let $A$ be a polarized abelian surface, and let $S$ be the Kummer surface attached to $A$. We view $S$ as the quotient of the blow-up $\widetilde{A}$ of $A$ along its 2 -torsion points by the involution induced by the multiplication-by- $(-1)$ map on $A$. In particular, since the cohomology of $\widetilde{A}$ differs from that of $A$ only by Hodge classes, we have the analogue of Theorem 2.12 for the very general polarized abelian surface as long as we allow the sum to run through all cycles $Q \in \mathrm{CH}^{n}\left(A^{m}\right)$ which are products of cycles of the form $\left(p_{i}\right)^{*} L,\left(p_{i}\right)^{*} E_{r},\left(p_{i, j}\right)^{*} P$, where $E_{r}$ denote the exceptional curves of $\widetilde{A}$. As a consequence, one can show that the conclusion of Theorem 1 holds for $\widetilde{A}$ by working with the universal polarized abelian surface of degree $d^{2}$ with level-4 structure. (We avoid working with level-2 structure in order to avoid having to deal with stacks.) In fact, quotienting by the action of multiplication-by- $(-1)$ fiber-wise, the conclusion of Theorem 1 holds for the induced universal family of Kummer surfaces.

Since $p r, p_{\wedge^{N}}$ and $p_{\wedge^{N-1}}$ are generically defined, arguing as in the proof of Proposition 2.13, we may construct a generically defined idempotent correspondence $q \in \mathrm{CH}^{2 N}\left(S^{N} \times S^{N}\right)$ such that

$$
q_{*} \mathrm{H}^{*}\left(S^{N}, \mathbb{Q}\right)=\operatorname{ker}\left(p r_{*}:\left(p_{\wedge^{N}}\right)_{*} \mathrm{H}^{*}\left(S^{N}, \mathbb{Q}\right) \rightarrow\left(p_{\wedge N-1}\right)_{*} \mathrm{H}^{*}\left(S^{N-1}, \mathbb{Q}\right)\right) .
$$

More precisely, there is a generically defined correspondence $\gamma$ on $S^{N-1} \times S^{N}$ such that $q=$ id $-\gamma \circ p_{\wedge N-1} \circ p r_{*} \circ p_{\wedge N}$. In particular, we see that

$$
q_{*} \mathrm{CH}_{l}\left(S^{N}\right) \supseteq \operatorname{ker}\left(p r_{*}:\left(p_{\wedge^{N}}\right)_{*} \mathrm{CH}_{l}\left(S^{N}\right) \rightarrow\left(p_{\wedge N-1}\right)_{*} \mathrm{CH}_{l}\left(S^{N-1}\right)\right) .
$$

On the other hand, defining $\pi_{S}^{2}$ to be the generically defined idempotent $\Delta_{S}-[0] \times S-S \times[0] \in$ $\mathrm{CH}^{2}(S \times S)$, we have

$$
\mathrm{CH}_{0}(S)_{\text {num }}=\left(\pi_{S}^{2}\right)_{*} \mathrm{CH}_{0}(S) .
$$

Therefore, in order to prove the theorem, it is enough to establish that

$$
\left(p_{\wedge N+1} \circ\left(q \otimes \pi_{S}^{2}\right)\right)_{*}\left(\mathrm{CH}_{l}\left(S^{N}\right) \otimes \mathrm{CH}_{0}(S)\right)=0 \text { for all } l<N .
$$

A cohomological calculation (as performed by Voisin [53, p. 274]) shows that

$$
\left(p_{\wedge N+1} \circ\left(q \otimes \pi_{S}^{2}\right)\right)^{*} \mathrm{H}^{i, j}\left(S^{N+1}, \mathbb{Q}\right)=0 \quad \text { for all } i<N .
$$

Therefore, by Theorem 1 applied to polarized abelian surfaces of degree $d^{2}$ with level-4 structure we obtain the stronger result that

$$
\left(p_{\wedge N+1} \circ\left(q \otimes \pi_{S}^{2}\right)\right)_{*} \mathrm{CH}_{l}\left(S^{N+1}\right)=0 \quad \text { for all } l<N .
$$

This concludes the proof of the theorem.

4.4. Varieties motivated by an abelian surface. Here, we say that a smooth projective variety is motivated by an abelian variety $A$ if its Chow motive is isomorphic to an object in the full, thick and rigid subcategory of Chow motives generated by $A$. In other words, $X$ is motivated by $A$ if $\mathfrak{h}(X)$ is isomorphic to a direct summand of a motive of the form $\bigoplus_{i} \mathfrak{h}\left(A^{m_{i}}\right)\left(n_{i}\right)$ for some integers $m_{i} \geq 0$ and $n_{i} \in \mathbb{Z}$. In particular, by Corollary 3.13, a strong form of the generalized Hodge conjecture holds for the powers of $X$; i.e.,

$$
\mathrm{N}_{H}^{r} \mathrm{H}^{k}\left(X^{m}, \mathbb{Q}\right)=\Gamma_{*} \mathrm{H}^{k-2 r}(B, \mathbb{Q}),
$$

where $B$ is a disjoint union of abelian varieties and where $\Gamma$ is a correspondence between $B$ and $X^{m}$. Examples of varieties motivated by an abelian surface include generalized Kummer varieties (see [57], and also [22, Corollary 6.3]). In particular, the following theorem applies to generalized Kummer varieties. 
Theorem 4.7. Let $X$ be a smooth projective variety of dimension d and let $\gamma \in \mathrm{CH}^{d}(X \times X)$ be a correspondence. Assume that the motive of $X$ is motivated by the motive of an abelian variety $A$ of dimension $\leq 2$. If $\gamma^{*} \mathrm{H}^{i, j}(X)=0$ for all $j<n$, then there exists an integer $N \geq 1$ such that $\left(\gamma^{\circ N}\right)_{*} \mathrm{CH}_{r}(X)=0$ for all $r<n$. In particular, if in addition $\gamma$ is an idempotent, then $\gamma_{*} \mathrm{CH}_{r}(X)=0$ for all $r<n$.

Proof. By Corollary 3.13, any Hodge sub-structure of $\mathrm{H}^{*}(X, \mathbb{Q})$ is a $L(A)$-sub-representation. One can then proceed as in the proof of Theorem 3.14 by invoking Proposition 3.12 to show that the cohomology class of $\gamma$ is a linear combination of cycles in $\mathrm{CH}^{*}(X \times X)$ that factor as

$$
\mathfrak{h}(X) \stackrel{\rho}{\longrightarrow} \mathfrak{h}(B)(n) \stackrel{\zeta}{\longrightarrow} \mathfrak{h}(X),
$$

for some abelian varieties $B$ and some correspondences $\rho$ and $\zeta$ that belong to $\mathrm{CH}^{*}(X \times B)$ and $\mathrm{CH}^{*}(B \times X)$, respectively. One concludes by Kimura finite-dimensionality as for instance in the proof of Theorem 2.15(1).

Remark 4.8. In the case where $X$ is a generalized Kummer variety, one can be more precise. By $[23, \S 4.5]$, one can define, for all integers $m \geq 0, \mathbb{Q}$-sub-algebras $\operatorname{DCH}^{*}\left(X^{m}\right) \subseteq \mathrm{CH}^{*}\left(X^{m}\right)$ consisting of distinguished cycles that map isomorphically to $\overline{\mathrm{CH}}^{*}\left(X^{m}\right)$ and that are compatible with pushforwards and pullbacks along projections. In particular, the composition of distinguished correspondences is distinguished. As such, in Theorem 4.7, if one chooses $\gamma$ to be a correspondence in $\mathrm{DCH}^{*}(X \times X)$ such that $\gamma^{*} \mathrm{H}^{i, j}(X)=0$ for all $j<n$, then Proposition 3.12 shows that $\gamma$ is a linear combination of cycles in $\mathrm{DCH}^{*}(X \times X)$ that factor as

$$
\mathfrak{h}(X) \stackrel{\rho}{\longrightarrow} \mathfrak{h}(B)(n) \stackrel{\zeta}{\longrightarrow} \mathfrak{h}(X),
$$

for some abelian varieties $B$ and some correspondences $\rho$ and $\zeta$ that belong to $\operatorname{DCH}^{*}(X \times B)$ and $\mathrm{DCH}^{*}(B \times X)$, respectively. One concludes that $\gamma_{*} \mathrm{CH}_{r}(X)=0$ for all $r<n$.

Remark 4.9. The results of Sections 4.2 and 4.3 could have been established by referring to Theorem 4.7 instead of Theorem 1. We chose to refer to Theorem 1 (which is concerned with generically defined cycles) because it is more elementary and does not appeal to Abdulali's theorem on the generalized Hodge conjecture for powers of CM abelian surfaces. Moreover the approach using generically defined cycles seems more natural and is probably better suited to adapt to other situations. Nonetheless, Theorem 4.10 below will use the full strength of Theorem 4.7.

4.5. Finite-order symplectomorphisms on generalized Kummer varieties. Let $(X, \omega)$ be a symplectic variety, that is, a smooth projective variety equipped with a nowhere degenerate 2-form $\omega$. A symplectomorphism of $(X, \omega)$ is an automorphism $f: X \rightarrow X$ such that $f^{*} \omega=\omega$. If $X$ is irreducible symplectic, it is expected as part of the Bloch conjectures that symplectomorphisms act unipotently on the Chow group of 0-cycles, and, due to the probable distinguishedness of symplectomorphisms in the sense of [23], it is in fact expected that symplectomorphisms act as the identity on the Chow group of 0-cycles. Most notably, this was established for symplectic involutions on K3 surfaces by Voisin [54] and extended to finite-order symplectomorphisms on K3 surfaces by Huybrechts [28]. This was also established for polarized symplectomorphisms of Fano varieties of lines on smooth cubic fourfolds by $\mathrm{Fu}$ [20], that is, for symplectomorphisms that preserve a given polarization. We extend that type of results to generalized Kummer varieties.

Theorem 4.10. Let $A$ be an abelian surface and let $f$ be a symplectomorphism of the generalized Kummer variety $K_{n}(A)$. Then $f_{*}: \mathrm{CH}_{0}\left(K_{n}(A)\right) \rightarrow \mathrm{CH}_{0}\left(K_{n}(A)\right)$ is unipotent. In particular, if $f$ is a finite-order symplectomorphism, then $f_{*}: \mathrm{CH}_{0}\left(K_{n}(A)\right) \rightarrow \mathrm{CH}_{0}\left(K_{n}(A)\right)$ is the identity.

Proof. Since $\mathrm{H}^{2 i, 0}\left(K_{n}(A)\right)=\mathrm{H}^{0}\left(\Omega_{K_{n}(A)}^{2 i}\right)=\mathbb{C} \omega^{i}$, and by definition of a symplectomorphism, $f^{*}$ acts as the identity on $\mathrm{H}^{2 i, 0}\left(K_{n}(A)\right)$ for all $i$. Therefore, by Theorem 4.7, $f$-id acts nilpotently on $\mathrm{CH}_{0}\left(K_{n}(A)\right)$. Suppose now that $f$ has finite order. In particular a positive power of $f$ acts 
as the identity on $\mathrm{CH}_{0}\left(K_{n}(A)\right)$. Since the gcd of the polynomials $X^{n}-1$ and $(X-1)^{N}$ is $X-1$, we find that $f$-id acts as zero on $\mathrm{CH}_{0}\left(K_{n}(A)\right)$.

Finally we note that if $f$ is a symplectomorphism of the generalized Kummer variety $K_{n}(A)$ induced by a symplectomorphism of $A$, then Pawar [42] showed that $f_{*}$ acts as the identity on $\mathrm{CH}_{0}\left(K_{n}(A)\right)_{(2 n)}$ (as defined in $\S 4.2$ ). We can extend Pawar's result and show that $f_{*}$ acts as the identity on the whole of $\mathrm{CH}_{0}\left(K_{n}(A)\right)$ :

Proposition 4.11. Suppose $f$ is a symplectomorphism of the generalized Kummer variety $K_{n}(A)$ induced by a symplectomorphism of $A$. Then $f_{*}$ acts as the identity on $\mathrm{CH}_{0}\left(K_{n}(A)\right)$.

Proof. One uses Remark 4.8 and notes that the graph of a symplectomorphism induced by a symplectomorphism of $A$ belongs to the sub-algebra $\mathrm{DCH}^{*}\left(K_{n}(A) \times K_{n}(A)\right)$ defined in [23, $\S 5.5]$.

\section{REFERENCES}

[1] Salman Abdulali, Hodge structures of CM-type, J. Ramanujan Math. Soc. 20 (2005), 155-162.

[2] Salman Abdulali, Tate twists of Hodge structures arising from abelian varieties of type IV, J. Pure Appl. Algebra 216 (2012), 1164-1170.

[3] Salman Abdulali, Tate twists of Hodge structures arising from abelian varieties, in Recent Advances in Hodge Theory: Period Domains, Algebraic Cycles, and Arithmetic (M. Kerr and G. Pearlstein, eds.), London Mathematical Society Lecture Note Series 427, Cambridge University Press, 2016, pp. 292-307.

[4] Jeff Achter, Sebastian Casalaina-Martin, S. and Charles Vial, Distinguished models of intermediate Jacobians, J. Inst. Math. Jussieu, to appear.

[5] Giuseppe Ancona, Décomposition du motif d'un schéma abélien universel, Ph.D. thesis, Université Paris 13, 2012, Thèse de doctorat Mathématiques, p. 60.

[6] Giuseppe Ancona, Décomposition de motifs abéliens, Manuscripta Math. 146 (2015), 307-328.

[7] Yves André, Pour une théorie inconditionnelle des motifs, Inst. Hautes Études Sci. Publ. Math. 83 (1996), 5-49.

[8] Yves André, Une introduction aux motifs (motifs purs, motifs mixtes, priodes), Panoramas et Synthèses 17. Société Mathématique de France, Paris, 2004.

[9] Arnaud Beauville, Variétés Kählériennes dont la première classe de Chern est nulle, J. Differential Geom. 18 (1983), 755-782.

[10] Arnaud Beauville, Quelques remarques sur la transformation de Fourier dans l'anneau de Chow d'une variété abélienne, Algebraic geometry (Tokyo/Kyoto, 1982), 238-260, Lecture Notes in Math., 1016, Springer, Berlin, 1983.

[11] Arnaud Beauville, Sur l'anneau de Chow d'une variété abélienne, Math. Ann. 273, 647-651 (1986).

[12] Christina Birkenhake and Herbert Lange, The dual polarization of an abelian variety, Arch. Math. (Basel) 73 (1999), 380-389.

[13] Spencer Bloch, Arnold Kas, David Lieberman, Zero cycles on surfaces with $p_{g}=0$, Compositio Math. 33 (1976), 135-145.

[14] Spencer Bloch and Vasudevan Srinivas, Remarks on correspondences and algebraic cycles, Amer. J. Math. 105 (1983), 1235-1253.

[15] Nicolas Bourbaki, Groupes et algèbres de Lie. Chapitres VII et VIII, Hermann, Paris 1975.

[16] Giuseppe Ceresa, $C$ is not algebraically equivalent to $C^{-}$in its Jacobian, Ann. of Math. 117 (1983), 285-291.

[17] Christopher Deninger and Jacob Murre, Motivic decomposition of abelian schemes and the Fourier transform, J. Reine Angew. Math. 422 (1991), 201-219.

[18] Eric Friedlander, Filtrations on algebraic cycles and homology, Ann. Sci. École Norm. Sup. 28 (1995), 317-343.

[19] Roberto Fringuelli and Roberto Pirisi, The Picard group of the universal abelian variety and the Franchetta conjecture for abelian varieties, arXiv:1603.09190.

[20] Lie Fu, On the action of symplectic automorphisms on the CH-groups of some hyper-Kähler fourfolds, Mathematische Zeitschrift 280 (2015), 307-334.

[21] Lie Fu, Robert Laterveer, and Charles Vial, The generalized Franchetta conjecture for some hyper-Kaehler varieties, J. Math. Pures Appl., to appear.

[22] Lie Fu, Zhiyu Tian, and Charles Vial, Motivic hyperKähler resolution conjecture: I. Generalized Kummer varieties, Geom. Topol. 23 (2019), 427-492.

[23] Lie Fu and Charles Vial, Distinguished cycles on varieties with motive of abelian type and the section property, J. Algebraic Geom., to appear. 2017.

[24] William Fulton and Joe Harris, Representation theory, A first course. Graduate Texts in Mathematics, 129. Springer-Verlag, New York, 1991. xvi+551 pp. 
[25] Fumio Hazama, Algebraic cycles on certain abelian varieties and powers of special surfaces, J. Fac. Sci. Univ. Tokyo Sect. IA Math. 31 (1985), 487-520.

[26] Fumio Hazama, The generalized Hodge conjecture for stably nondegenerate abelian varieties, Compositio Math. 93 (1994), 129-137.

[27] Fumio Hazama, On the general Hodge conjecture for abelian varieties of CM-type, Publ. Res. Inst. Math. Sci. 39 (2003), 625-655.

[28] Daniel Huybrechts, Symplectic automorphisms of K3 surfaces of arbritrary finite order, Math. Res. Lett. 19 (2012), 947-951.

[29] Shun-Ichi Kimura, Chow groups are finite dimensional, in some sense, Math. Ann. 331 (2005), $173-201$.

[30] Steven Kleiman, Algebraic cycles and the Weil conjectures, Dix exposés sur la cohomologie des schémas, 359-386, Adv. Stud. Pure Math., 3, North-Holland, Amsterdam, 1968.

[31] Robert Laterveer, Some results on a conjecture of Voisin for surfaces of geometric genus one, Boll. Unione Mat. Italiana, to appear.

[32] Robert Laterveer, Some desultory remarks concerning algebraic cycles and Calabi-Yau threefolds, Rend. Circ. Mat. Palermo, to appear.

[33] Hsueh-Yung Lin, On the Chow group of zero-cycles of a generalized Kummer variety, Adv. Math. 298 (2016), 448-472.

[34] Hsueh-Yung Lin, Corrigendum to "On the Chow group of zero-cycles of a generalized Kummer variety", Adv. Math. 331 (2018), 1016-1021.

[35] James Milne, Lefschetz classes on abelian varieties, Duke Math. J. 96 (1999), 639-675.

[36] Ben Moonen and Yuri Zarhin, Hodge classes on abelian varieties of low dimension, Math. Ann. 315 (1999), 711-733.

[37] Ben Moonen, On the Chow motive of an abelian scheme with non-trivial endomorphisms, J. Reine Angew. Math. 711 (2016), 75-109. MR 3456759

[38] David Mumford, John Fogarty, Frances Kirwan, Geometric invariant theory, Third edition. Ergebnisse der Mathematik und ihrer Grenzgebiete, 34. Springer-Verlag, Berlin, 1994. xiv+292 pp.

[39] Kumar Murty, Exceptional Hodge classes on certain abelian varieties, Math. Ann. 268 (1984), 197-206.

[40] Madhav Nori, Cycles on the generic abelian threefold, Proc. Indian Acad. Sci. Math. Sci. 99 (1989), 191-196.

[41] Peter O'Sullivan, Algebraic cycles on an abelian variety, J. Reine Angew. Math. 654 (2011), 1-81.

[42] Rakesh Pawar, Action of correspondences on filtrations on cohomology and 0-cycles of abelian varieties, Math. Z. 292 (2019), 655-675.

[43] Kenneth Ribet, Hodge classes on certain types of abelian varieties, Amer. J. Math. 105 (1983), 523-538.

[44] Mingmin Shen and Charles Vial, The Fourier transform for certain hyperKähler fourfolds, Mem. Amer. Math. Soc. 240 (2016), no. 1139, vii+163 pp.

[45] Alexander Shermenev, Motif of an Abelian variety, Funckcional. Anal. i Prilozen 8 (1974), 5561.

[46] Goro Shimura, On analytic families of polarized abelian varieties and automorphic functions, Ann. of Math. 781963 149-192.

[47] Tony Scholl, Classical motives, Motives (Seattle, WA, 1991), 163-187, Proc, Sympos. Pure Math., 55, Part $1,1994$.

[48] S. G. Tankeev, Cycles on simple abelian varieties of prime dimension, Izv. Akad. Nauk SSSR Ser. Mat. 46 (1982), 155-170, 192.

[49] S. G. Tankeev, Abelian varieties and the general Hodge conjecture, Izv. Ross. Akad. Nauk Ser. Mat. 57 (1993), 192-206.

[50] Charles Vial, Niveau and coniveau filtrations on cohomology groups and Chow groups, Proc. Lond. Math. Soc. 106 (2013), 410-444.

[51] Vladimir Voevodsky, A nilpotence theorem for cycles algebraically equivalent to zero, Internat. Math. Res. Notices 1995, 187-198.

[52] Claire Voisin, Sur les zéro-cycles de certaines hypersurfaces munies d'un automorphisme, Ann. Scuola Norm. Sup. Pisa Cl. Sci. (4) 19 (1992), 473-492.

[53] Claire Voisin, Remarks on zero-cycles of self-products of varieties, Moduli of vector bundles (Sanda, 1994; Kyoto, 1994), 265-285, Lecture Notes in Pure and Appl. Math., 179, Dekker, New York, 1996.

[54] Claire Voisin, Symplectic involutions of K3 surfaces act trivially on $\mathrm{CH}_{0}$, Doc. Math. 17 (2012), 851-860.

[55] Claire Voisin, Bloch's conjecture for Catanese and Barlow surfaces, J. Differential Geom. 97 (2014), 149175.

[56] Claire Voisin, Chow rings, decomposition of the diagonal, and the topology of families, Annals of Mathematics Studies, 187. Princeton University Press, Princeton, NJ, 2014. viii+163 pp.

[57] Ze Xu, Algebraic cycles on a generalized Kummer variety, Int. Math. Res. Not. IMRN 2018, 932-948.

Universität Bielefeld, Germany

E-mail address: vial@math.uni-bielefeld.de 